\title{
Gradient theory computation of the radius-dependent surface tension and nucleation rate for n-nonane clusters
}

Citation for published version (APA):

Hruby, J., Labetski, D. G., \& Dongen, van, M. E. H. (2007). Gradient theory computation of the radius-dependent surface tension and nucleation rate for n-nonane clusters. Journal of Chemical Physics, 127(16), 164720-1/14. https://doi.org/10.1063/1.2799515

DOI:

10.1063/1.2799515

Document status and date:

Published: 01/01/2007

Document Version:

Publisher's PDF, also known as Version of Record (includes final page, issue and volume numbers)

Please check the document version of this publication:

- A submitted manuscript is the version of the article upon submission and before peer-review. There can be important differences between the submitted version and the official published version of record. People interested in the research are advised to contact the author for the final version of the publication, or visit the $\mathrm{DOI}$ to the publisher's website.

- The final author version and the galley proof are versions of the publication after peer review.

- The final published version features the final layout of the paper including the volume, issue and page numbers.

Link to publication

\section{General rights}

Copyright and moral rights for the publications made accessible in the public portal are retained by the authors and/or other copyright owners and it is a condition of accessing publications that users recognise and abide by the legal requirements associated with these rights.

- Users may download and print one copy of any publication from the public portal for the purpose of private study or research.

- You may not further distribute the material or use it for any profit-making activity or commercial gain

- You may freely distribute the URL identifying the publication in the public portal.

If the publication is distributed under the terms of Article 25fa of the Dutch Copyright Act, indicated by the "Taverne" license above, please follow below link for the End User Agreement:

www.tue.nl/taverne

Take down policy

If you believe that this document breaches copyright please contact us at:

openaccess@tue.nl

providing details and we will investigate your claim. 


\title{
Gradient theory computation of the radius-dependent surface tension and nucleation rate for $n$-nonane clusters
}

\author{
J. Hrubýa) \\ Institute of Thermomechanics AS CR, v.v.i., Dolejškova 5, CZ-18200 Prague 8, Czech Republic \\ D. G. Labetski and M. E. H. van Dongen \\ Department of Applied Physics, Eindhoven University of Technology, P.O. Box 513, 5600 MB Eindhoven, \\ The Netherlands
}

(Received 15 June 2007; accepted 24 September 2007; published online 30 October 2007)

\begin{abstract}
The Van der Waals-Cahn-Hilliard gradient theory (GT) is applied to determine the structure and the work of formation of clusters in supersaturated $n$-nonane vapor. The results are analyzed as functions of the difference of pressures of the liquid phase and vapor phase in chemical equilibrium, which is a measure for the supersaturation. The surface tension as a function of pressure difference shows first a weak maximum and then decreases monotonically. The computed Tolman length is in agreement with earlier results [L. Gránásy, J. Chem. Phys. 109, 9660 (1998)] obtained with a different equation of state. A method based on the Gibbs adsorption equation is developed to check the consistency of GT results (or other simulation techniques providing the work of formation and excess number of molecules), and to enable an efficient interpolation. A cluster model is devised based on the density profile of the planar phase interface. Using this model we analyze the dependency of the surface tension on the pressure difference. We find three major contributions: (i) the effect of asymmetry of the density profile resulting into a linear increase of the surface tension, (ii) the effect of finite thickness of the phase interface resulting into a negative quadratic term, and (iii) the effect of buildup of a low-density tail of the density profile, also contributing as a negative quadratic term. Contributions (i)-(iii) fully explain the dependency of the surface tension on the pressure difference, including the range relevant to nucleation experiments. Contributions (i) and (ii) can be predicted from the planar density profile. The work of formation of noncritical clusters is derived and the nucleation rate is computed. The computed nucleation rates are closer to the experimental nucleation rate results than the classical Becker-Döring theory, and also the dependence on supersaturation is better predicted. () 2007 American Institute of Physics.
\end{abstract}

[DOI: 10.1063/1.2799515]

\section{INTRODUCTION}

The classical theory of nucleation of droplets from supersaturated vapors has been elaborated by Becker and Döring ${ }^{1}$ and others. One of the fundamentals of this theory is modeling the cluster as a droplet with a sharp phase interface. In reality, the thickness of the phase interface is often comparable to the radius of the droplet. Possibly, the cluster may not even possess a core identifiable with the bulk liquid phase. It is, therefore, more realistic to consider a diffuse interface model, in which the density varies continuously across the phase interface. Such a model has been devised already by Van der Waals. ${ }^{2}$ In this model, the local Helmholtz energy density not only depends on the local molar density and temperature, but also on the molar density gradient. Several interesting applications of the density gradient theory have been elaborated by Cahn and Hilliard. ${ }^{3}$ The last authors also first applied the gradient theory to nucleation. ${ }^{4}$ Theoretical foundations of the gradient theory and the related statistical physics background are given in the books by Rowlinson and Widom, ${ }^{5}$ Davis, ${ }^{6}$ and Kalikmanov. ${ }^{7}$ A recent

${ }^{a)}$ Electronic mail: hruby@it.cas.cz study of the gradient theory with comparison to molecular simulations was given by Baidakov et al. ${ }^{8}$ The gradient theory is closely related to the density functional theory, first applied to gas-liquid nucleation by Oxtoby and Evans. ${ }^{9}$ Both theories are based on the mean-field approximation. The gradient theory can be easily related to the classical capillarity approach. This relation is facilitated by the fact that both approaches are based on an equation of state-in the capillarity theory the equation of state is only used for the homogeneous liquid and gas regions, while in the gradient theory one single equation of state is used for the whole system, including the phase interface. The value of the capillarity theory is that it provides a simple mathematical framework and it is evidently correct for macroscopic droplets, and its weakness being that it does not give any advice concerning the dependency of the surface tension on the curvature of the phase interface. The dependency of the surface tension on curvature was first studied by Tolman, ${ }^{10}$ and semiphenomenological models were developed among others by Dillmann and Meier, ${ }^{11}$ Delale and Meier, ${ }^{12}$ and Laaksonen et al. ${ }^{13}$ A novel nucleation theory introducing a concept of microscopic surface tension was recently proposed by Kalikmanov. $^{14}$ 
This work represents a continuation of a long-term research of nucleation in natural gas. ${ }^{15-17} n$-nonane represents heavy hydrocarbons dissolved in the raw natural gas. In this study, pure $n$-nonane is considered as an initial point for the analysis of nucleation in mixtures of $n$-nonane and methane at high pressures where a peculiar behavior was observed. ${ }^{18,19}$ The properties of $n$-nonane are well suited for nucleation experiments. Nucleation rate data are available and are obtained using expansion cloud chambers, ${ }^{20-22}$ static diffusion cloud chambers, ${ }^{23-27}$ and an expansion wave tube. ${ }^{17,28}$ Numerous theoretical studies concerning $n$-nonane nucleation exist. Nyquist et al. ${ }^{29}$ computed the work of formation of critical $n$-nonane clusters using density functional theory with either the Yukawa potential and the LennardJones potential. They found that the two computations almost overlapped. Gránásy ${ }^{30,31}$ computed the Tolman length for $n$-nonane represented with the van der Waals equation of state using the gradient theory. Barrett ${ }^{32}$ compared density functional computations with gradient theory computations based on the Peng-Robinson ${ }^{33}$ equation of state and on the van der Waals equation of state with the Carnahan-Starling ${ }^{34}$ repulsive term. Barrett ${ }^{35}$ also developed a correction of the nucleation rates computed with density functional theories for inclusion of the cluster translation. Natarajan et al. ${ }^{36}$ studied evaporation of $n$-nonane clusters by molecular dynamics. They also provided instructive snapshots of simulated clusters of sizes similar to those treated in the present work.

The paper is organized as follows. In Sec. II we give an outline of the Gibbsian droplet model and we introduce the gradient theory. Both concepts are needed because a focus of the paper is to interpret the gradient theory results within the Gibbsian framework. In Sec. III we provide the results of the gradient theory computations and develop a thermodynamics-based tool to check the internal consistency of the computations and to accurately interpolate the results. In Sec. IV we develop a model of a cluster based on the density profile of the planar phase interface. We identify several contributions to the dependency of the surface tension on the pressure difference (or supersaturation). In Sec. V we derive the work of formation of noncritical clusters and provide an expression for the nucleation rate. We compare the nucleation rates with available nucleation rate data and with the classical nucleation theory. In Sec. VI we draw conclusions. Some lengthier derivations are given in Appendix A, and a summary of thermophysical properties is given in Appendix B.

\section{THEORY}

\section{A. Gibbsian droplet model}

One assumes that the interior of the cluster-modeled as a microscopic droplet-is formed by a liquid at pressure $p_{L}$, which is higher than the pressure $p_{G}$ of the surrounding gas phase due to the Laplace pressure,

$$
p_{L}-p_{G} \equiv \Delta p=\frac{2 \sigma}{r_{s}}
$$

where $\sigma$ is the surface tension and $r_{s}$ is the radius of the droplet. More accurately, $r_{s}$ is the radius of the sphere of tension. ${ }^{5}$ Only for this radius definition the Laplace equation can be written in the simple form of Eq. (1). Of special importance in the classical nucleation theory is the critical cluster. The radius of the critical cluster is such that the probabilities of growing and shrinking are equal. In thermodynamic language this translates to the equality of chemical potentials of the liquid and gas phases,

$$
\mu_{L}\left(p_{L}, T\right)=\mu_{G}\left(p_{G}, T\right) .
$$

In a supersaturated vapor the gas pressure $p_{G}$ is greater than $p_{\infty}$, the pressure of the saturated vapor above a flat phase interface $\left(r_{s} \rightarrow \infty\right)$. Then, Eq. (2) gives a liquid pressure $p_{L}$ greater than $p_{\infty}$, and a positive pressure difference $\Delta p$. The computation is done using an equation of state valid for bulk liquid and vapor (here, the Peng-Robinson equation of state ${ }^{33}$ ). The liquid density $\rho_{L}$ corresponds to a compressed bulk liquid at pressure $p_{L}$. This provides a realistic picture for large clusters: Allowing for the liquid compressibility in the classical nucleation theory brings a significant improvement. ${ }^{37}$ However, if the cluster is so small that the thickness of the phase interface is similar to the cluster radius, the density at the center of the cluster does not reach the bulk liquid value $\rho_{L}$. Despite that, we keep on using $p_{L}$ obtained from Eq. (2) and the corresponding $\rho_{L}$ in deriving the surface tension and related quantities from the results of the gradient theory, literally maintaining the Gibbsian framework. The pressure difference $\Delta p$ will serve as a suitable measure of supersaturation.

If the surface tension is known, the work of formation $\Delta \Omega$ of the critical cluster can be computed as

$$
\Delta \Omega=\frac{1}{3} \sigma A_{s},
$$

where $A_{s}=4 \pi r_{s}^{2}$ now is the surface area of the (critical) droplet. An inherent part of the classical nucleation theory is the so-called capillarity approximation: We approximate the actual surface tension of the cluster with the surface tension of the flat phase interface at thermodynamic equilibrium, a quantity which can be found in tables. Accepting the capillarity approximation, the set of equations, Eqs. (1)-(3), is complete and we can compute the work of formation.

In the present work we go beyond the capillarity approximation. Using the gradient theory we will directly compute the work of formation $\Delta \Omega$ for given conditions of the gas phase. Then, using Eqs. (1) and (3), we compute the corresponding radius and surface tension,

$$
\begin{aligned}
& r_{s}=\left(\frac{3 \Delta \Omega}{2 \pi \Delta p}\right)^{1 / 3}, \\
& \sigma=\left(\frac{3 \Delta \Omega \Delta p^{2}}{16 \pi}\right)^{1 / 3} .
\end{aligned}
$$


The gradient theory also allows computation of the excess number of molecules $\Delta N$. In the Gibbsian picture the excess number of molecules is given as

$$
\Delta N=V_{s} \Delta \rho+A_{s} \Gamma,
$$

where $V_{s}=\frac{4}{3} \pi r_{s}^{3}$ is the volume of the sphere of tension, $\Delta \rho$ $\equiv \rho_{L}-\rho_{G}$, and $\Gamma$ is the surface excess of molecules or adsorption. Equation (6) will be used to extract the adsorption $\Gamma$ from gradient theory computations. From the differential analysis of the equations defining the droplet model it follows that the surface tension and adsorption are related by the Gibbs adsorption equation, ${ }^{5}$

$$
\mathrm{d} \sigma=-\Gamma \mathrm{d} \mu .
$$

This equation is valid for isothermal changes of the critical cluster and it is used such that Eq. (2) is satisfied along the path of differentiation.

Alternatively, the droplet can be modeled as a discontinuous profile without surface excess, thus defining the socalled equimolar surface and equimolar radius $r_{e}$,

$$
\Delta N=\frac{4}{3} \pi r_{e}^{3} \Delta \rho .
$$

The difference of the two radii is the Tolman ${ }^{10}$ length,

$$
\delta \equiv r_{e}-r_{s} .
$$

The importance of the Tolman length is in its relation to the dependence of the surface tension on the radius. In the present work we discuss rather the dependence on the pressure difference $\Delta p$. [Both are related by Eq. (1).] Using the isothermal Gibbs-Duhem equations for both phases we derive

$$
\mathrm{d} \mu=\frac{\mathrm{d} \Delta p}{\Delta \rho} .
$$

Substituting this expression into the Gibbs adsorption equation [Eq. (7)] we find

$$
\mathrm{d} \sigma=-t \mathrm{~d} \Delta p,
$$

where

$$
t \equiv \Gamma / \Delta \rho
$$

has a dimension of length. For a future reference we relate $t$ directly to the excess number of molecules $\Delta N$. Combining Eqs. (6) and (12), we have

$$
t=\frac{1}{A_{s}}\left(\frac{\Delta N}{\Delta \rho}-V_{s}\right) .
$$

The lengths $\delta$ and $t$ can be related by comparing Eqs. (6) and (8),

$$
t=\delta+\frac{\delta^{2}}{r_{s}}+\frac{\delta^{3}}{3 r_{s}^{2}} .
$$

For the planar interface $\left(r_{s} \rightarrow \infty\right)$ these lengths are equal: $t_{\infty}$ $=\delta_{\infty}$.

\section{B. Gradient theory}

The gradient theory ${ }^{2-4}$ operates with continuous density profiles $\rho(r)$. The local Helmholtz energy density of an inhomogeneous fluid comprises two terms,

$$
\Phi=\Phi_{\mathrm{hom}}(\rho)+\frac{1}{2} c\left(\frac{\mathrm{d} \sigma}{\mathrm{d} r}\right)^{2} .
$$

The first term is the Helmholtz energy density of a homogeneous fluid at the actual local density. The second term is a correction for inhomogeneity. This is represented by the gradient square term and a positive coefficient $c$, called the influence parameter. In this work the influence parameter is taken as independent of density. The influence parameter can be related to the direct correlation function of the homogeneous fluid. This approach is not practical for nonspherical molecules. Rather, the influence parameter is obtained by matching the experimental value of the surface tension of the flat phase interface as discussed later. The chemical potential is homogeneous throughout the system. Therefore, the grand potential density is given as

$$
\omega=\Phi-\rho \mu_{G} .
$$

In the homogeneous gas phase, the grand potential density reduces to $-p_{G}$. The work of formation of a cluster can be expressed as a difference between the grand potential of a system containing the cluster and the gas phase and the grand potential of the system containing the gas phase only,

$$
\Delta \Omega=\int_{0}^{\infty}\left[\Delta \omega_{\text {hom }}(\rho)+\frac{1}{2} c\left(\frac{\mathrm{d} \rho}{\mathrm{d} r}\right)^{2}\right] 4 \pi r^{2} \mathrm{~d} r,
$$

where the excess grand potential density $\Delta \omega_{\text {hom }}$ is given as

$$
\Delta \omega_{\text {hom }}(\rho) \equiv \Phi_{\text {hom }}(\rho)-\rho \mu_{G}+p_{G} .
$$

The function $\Delta \omega_{\text {hom }}(\rho)$ is obtained from a suitable equation of state of the fluid. The surface tension of the flat interface can be obtained in a very similar fashion,

$$
\sigma_{\infty}=\int_{-\infty}^{\infty}\left[\Delta \omega_{\text {hom }}(\rho)+\frac{1}{2} c\left(\frac{\mathrm{d} \rho}{\mathrm{d} z}\right)^{2}\right] \mathrm{d} z,
$$

where $z$ is a coordinate perpendicular to the phase interface. To be able to compute the work of formation of a cluster using Eq. (18), the density profile $\rho(r)$ is required.

For the flat surface, the density profile is found by utilizing the general principle that a thermodynamic system of given volume, temperature, and chemical potential will equilibrate in a state of lowest grand potential. The task is to find a density profile minimizing integral [Eq. (19)]. The necessary condition is that the profile is stationary, i.e., for small perturbations around this profile the value of the integral will not change. This functional problem can be reduced to Euler-Lagrange equation,

$$
\frac{\mathrm{d}^{2} \rho}{\mathrm{d} z^{2}}=\frac{1}{c} \Delta \mu(\rho),
$$

where 


$$
\Delta \mu \equiv \frac{\partial \Delta \omega_{\mathrm{hom}}}{\partial \rho}=\mu(\rho)-\mu_{G} .
$$

Equation (20) is to be solved for boundary conditions $\rho(-\infty)=\rho_{L}$ and $\rho(\infty)=\rho_{G}$, where $\rho_{L}$ and $\rho_{G}$ are densities of the homogeneous liquid $(L)$ and gaseous $(G)$ phases in thermodynamic equilibrium, determined by equating the pressures and chemical potentials. A first integral of Eq. (20) can easily be found yielding expressions for the density profile and surface tension,

$$
\begin{aligned}
& z=\int_{\rho(0)}^{\rho(z)}\left[\frac{c}{2 \Delta \omega_{\mathrm{hom}}(\rho)}\right]^{1 / 2} \mathrm{~d} \rho, \\
& \sigma_{\infty}=(2 c)^{1 / 2} \int_{\rho_{G}}^{\rho_{L}}\left[\Delta \omega_{\mathrm{hom}}(\rho)\right]^{1 / 2} \mathrm{~d} \rho .
\end{aligned}
$$

The density profile $\rho(r)$ of the critical cluster will not minimize the grand potential, because the critical cluster is in labile (not stable) equilibrium with the gas phase. The critical density profile corresponds with a saddle point of the grand potential. Roughly speaking, it is a minimum of the grand potential with respect to variations of the shape of the cluster, and a maximum with respect to variations of its radius. This problem has been discussed by Oxtoby and Evans ${ }^{9}$ and by Barrett. ${ }^{35}$ In the gradient theory it is possible to solve both the planar and the spherical cases by differential EulerLagrange equations. For the critical cluster, the EulerLagrange equation corresponding to the functional (17) is

$$
\frac{\mathrm{d}^{2} \rho}{\mathrm{d} r^{2}}+\frac{2}{r} \frac{\mathrm{d} \rho}{\mathrm{d} r}=\frac{1}{c} \Delta \mu(\rho) .
$$

Equation (24) must be solved numerically for boundary conditions $\rho(r \rightarrow \infty)=\rho_{G}$ and $(\mathrm{d} \rho / \mathrm{d} r)(r=0)=0$.

The above given equations are sufficient for the computation of the density profiles, the planar surface tension, and the work of formation of clusters. In addition, we mention formulas which proved useful in assessing the numerical accuracy of the computed density profiles. Let us assume that a profile $\rho(z)$ satisfies the Euler-Lagrange equation [Eq. (20)]. We use the chain rule $\left(\mathrm{d} \Delta \omega_{\text {hom }} / \mathrm{d} z\right)=\Delta \mu \cdot(\mathrm{d} \rho / \mathrm{d} z)$. Then, with Eq. (20) we obtain $\mathrm{d} \Delta \omega_{\text {hom }} / \mathrm{d} z=\frac{1}{2} c\left[\mathrm{~d}(\mathrm{~d} \rho / \mathrm{d} z)^{2} / \mathrm{d} z\right]$. By integration and substitution in Eq. (19), we find

$$
\sigma_{\infty}=\int_{-\infty}^{\infty} c\left(\frac{\mathrm{d} \rho}{\mathrm{d} z}\right)^{2} \mathrm{~d} z .
$$

Using this result, the density gradient can be eliminated from Eq. (19). In this way we obtain

$$
\sigma_{\infty}=2 \int_{-\infty}^{\infty} \Delta \omega_{\text {hom }} \mathrm{d} z .
$$

In Eq. (25) the surface tension only depends on the density gradient. On the other hand, in Eq. (26) the surface tension is given as an integral of a function of density. Comparison of the planar surface tensions computed using these two formulas is an efficient test of the numerical accuracy of the computed profile $\rho(z)$.
Formulas analogous to Eqs. (25) and (26) for $\sigma_{\infty}$ can be derived for the work of formation of the critical cluster,

$$
\begin{aligned}
& \Delta \Omega=\frac{1}{3} \int_{0}^{\infty} c\left(\frac{\mathrm{d} \rho}{\mathrm{d} r}\right)^{2} 4 \pi r^{2} \mathrm{~d} r, \\
& \Delta \Omega=-2 \int_{0}^{\infty} \Delta \omega_{\mathrm{hom}} 4 \pi r^{2} \mathrm{~d} r .
\end{aligned}
$$

These equations have a practical importance for checking the accuracy of gradient theory computations. The derivation of Eqs. (27) and (28) follows about the same line as for the planar case but requires some additional labor. We give the derivation in Appendix A. Another important output of the gradient theory computations is the excess number of molecules. For the continuous profile it is computed straightforwardly as [cf. Eq. (6)]

$$
\Delta N=\int_{0}^{\infty}\left[\rho(r)-\rho_{G}\right] 4 \pi r^{2} \mathrm{~d} r .
$$

The limit $r_{s} \rightarrow \infty$ (planar phase interface) deserves special attention. The Tolman length for the planar phase interface can be expressed as

$$
\delta_{\infty}=z_{e}-z_{s},
$$

where $z_{e}$ is the position (height) of the equimolar surface (a horizontal plane), and $z_{s}$ is the position of the surface of tension. Provided that the liquid phase exists at $z \rightarrow-\infty$ and the gas phase exists at $z \rightarrow \infty$, the equimolar surface is at a position

$$
z_{e}=\frac{1}{\rho_{L}-\rho_{G}}\left\{\int_{-\infty}^{0}\left[\rho(z)-\rho_{L}\right] \mathrm{d} z+\int_{0}^{\infty}\left[\rho(z)-\rho_{G}\right] \mathrm{d} z\right\} .
$$

The position of the equimolar surface obtained in this way represents a limiting case of the equimolar surface of a spherical cluster introduced in Eq. (8). The position of the surface of tension with respect to the same coordinate system is computed based on the first moment of the gradient square term,

$$
z_{s}=\frac{1}{\sigma_{\infty}} \int_{-\infty}^{\infty} c\left(\frac{\mathrm{d} \rho}{\mathrm{d} z}\right)^{2} z \mathrm{~d} z .
$$

Derivation of this formula is more difficult. The proof is given in Appendix A along with some other derivations.

\section{RESULTS OF THE GRADIENT THEORY COMPUTATIONS}

The gradient theory computations were based on the Peng-Robinson equation of state. ${ }^{33}$ The influence parameter was obtained by fitting the orthobaric surface tension data for $n$-nonane by Jasper et al. ${ }^{38}$ The influence parameter was computed using Eq. (23) valid for the planar phase interface. The experimental surface tension data were represented within their accuracy using a constant (temperatureindependent) influence parameter $c=3.031 \times 10^{-66} \mathrm{~J} \mathrm{~m}^{5}$. 


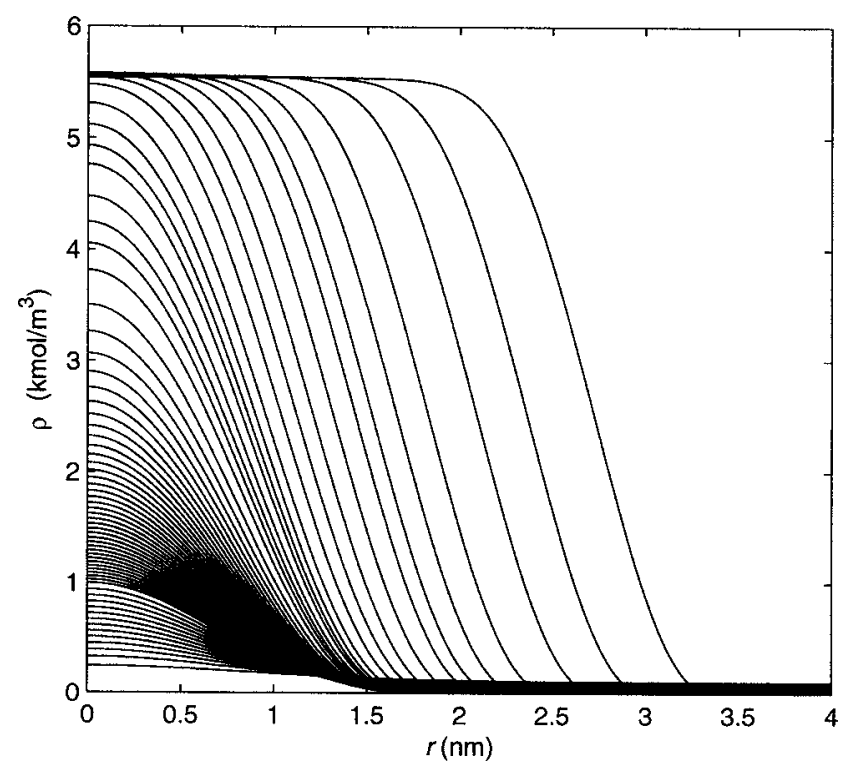

FIG. 1. Density profiles of $n$-nonane clusters at $230 \mathrm{~K}$ computed with the gradient theory. Profiles are computed for vapor pressure multiples $p_{G} / p_{\text {sat }, \mathrm{PR}}=8,11,15,22,30,40,50,70,100,140,200,300,400,500,600,800$, 1000, 1200, 1500, 2000,...,19000, 19500, 20000, 21000, 32000, 33000.

The gradient theory computations of critical clusters were performed at $230 \mathrm{~K}$. This isotherm was chosen because of the availability of experimental nucleation rate data. The corresponding vapor pressure computed from the PengRobinson equation of state was $p_{\text {sat } P R}=3.204 \mathrm{~Pa}$. We computed 69 density profiles for vapor pressures ranging from $8 p_{\text {sat,PR }}$ to $33000 p_{\mathrm{PR}}$. The profiles are shown in Fig. 1. For pressures smaller than $8 p_{\text {sat,PR }}$ the iteration did not reach our convergence criterion (discussed below). From above, the pressure range was limited by the vapor-liquid spinodal at 1.080 bar. The computation has been carried out using the BVP4 solver in MATLAB.

Figure 2 shows the works of formation computed as an arithmetic average of formulas (27) and (28). Figure 3 shows the excess numbers of molecules, computed using Eq. (29).

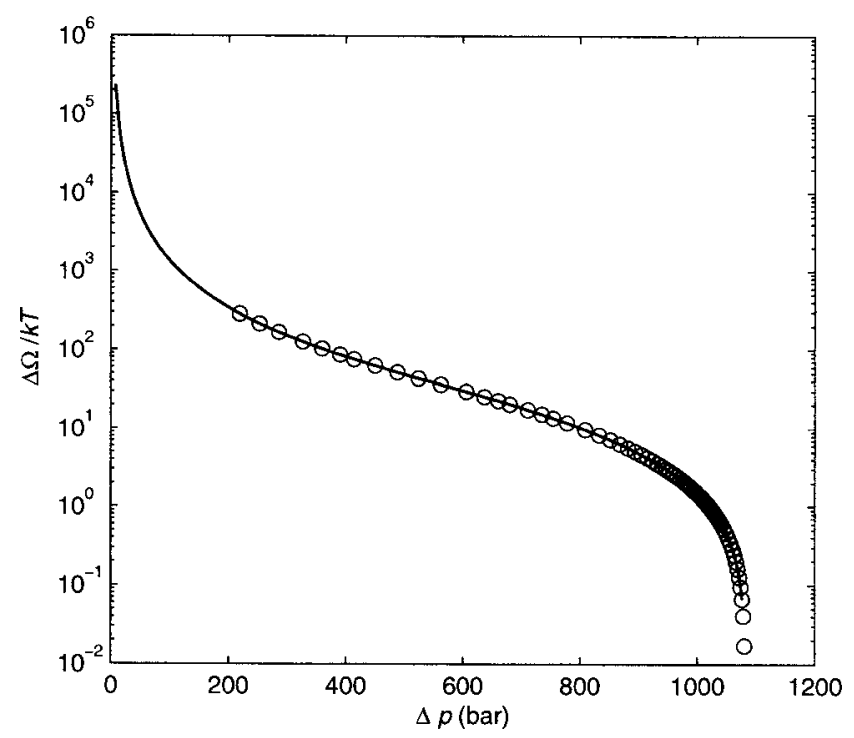

FIG. 2. The work of formation $\Delta \Omega$ of $n$-nonane clusters at $230 \mathrm{~K}$ as a function of the pressure difference $\Delta p$. The solid line is based on Eq. (33).

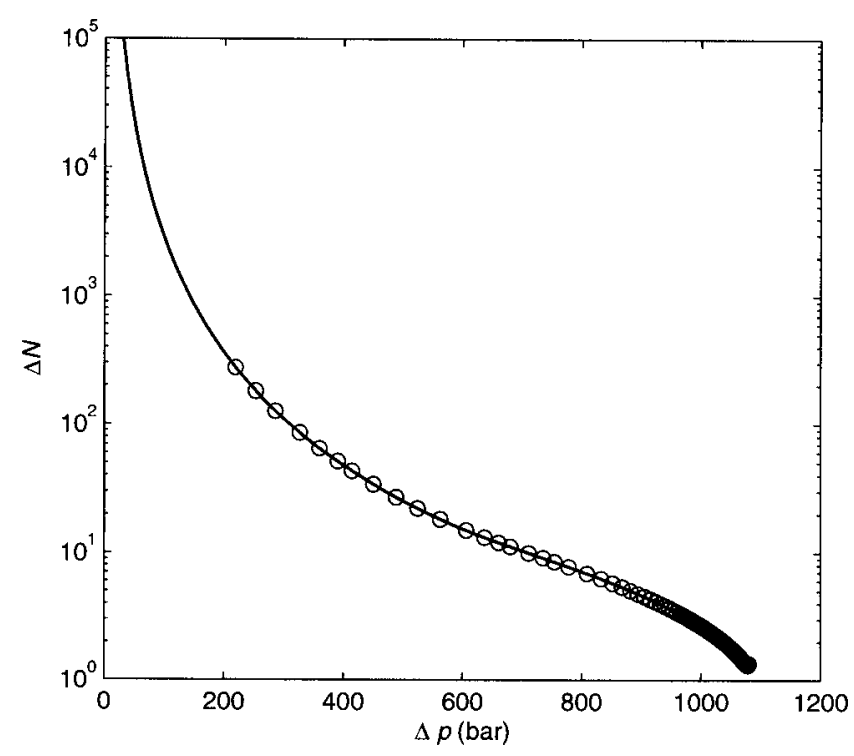

FIG. 3. Excess number of molecules $\Delta N$ of $n$-nonane clusters at $230 \mathrm{~K}$ as a function of the pressure difference $\Delta p$. The solid line is based on Eq. (33).

$\Delta N$ is a monotonically decreasing function of $\Delta p$ except very close to the spinodal where it reaches a minimum and increases again, finally diverging at $\Delta p=1081$ bars, where the spinodal is located. Close to the spinodal, a similarity solution of the Euler-Lagrange equation [Eq. (24)] can be developed as shown by Cahn and Hilliard ${ }^{4}$ and in more detail by Wilemski and $\mathrm{Li}^{39}$ This is a region of diffuse density fluctuations. The resulting profiles (see Fig. 1) are rather an artifact of the mean-field approximation. The clusters relevant to nucleation (at least in this study) are on the decreasing branch of the $\Delta N$ curve.

Figure 4 shows the surface tension computed using Eq. (5) from the previously determined works of formation [Eqs. (27) and (28)]. The difference of the surface tensions computed with these formulas was used as an indicator for terminating the iterative computation of the density profiles, by

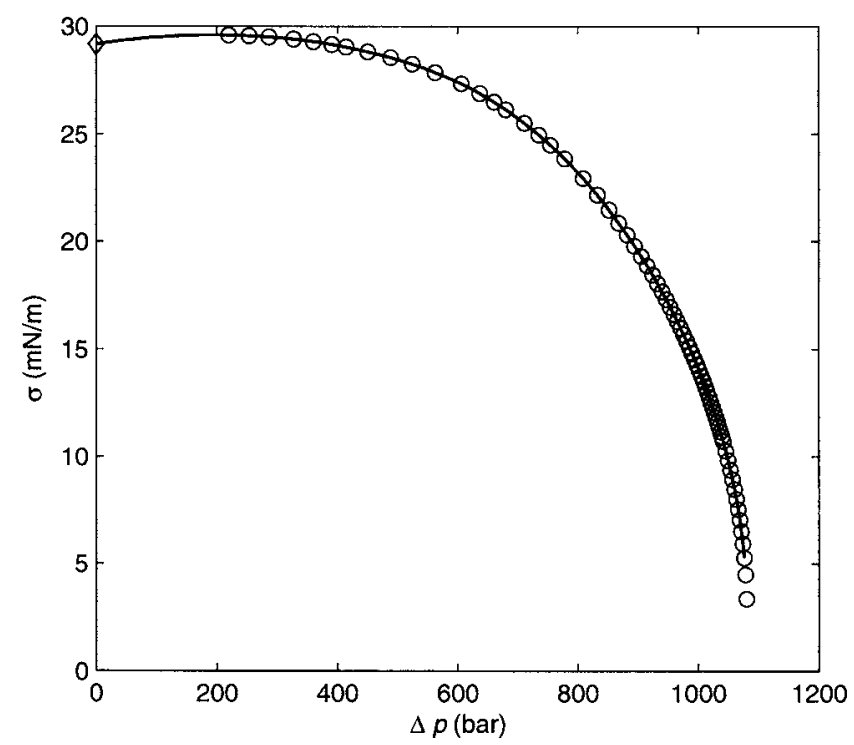

FIG. 4. Surface tension of $n$-nonane clusters at $230 \mathrm{~K}$ as a function of the pressure difference $\Delta p$. The solid line is given by Eq. (33). 


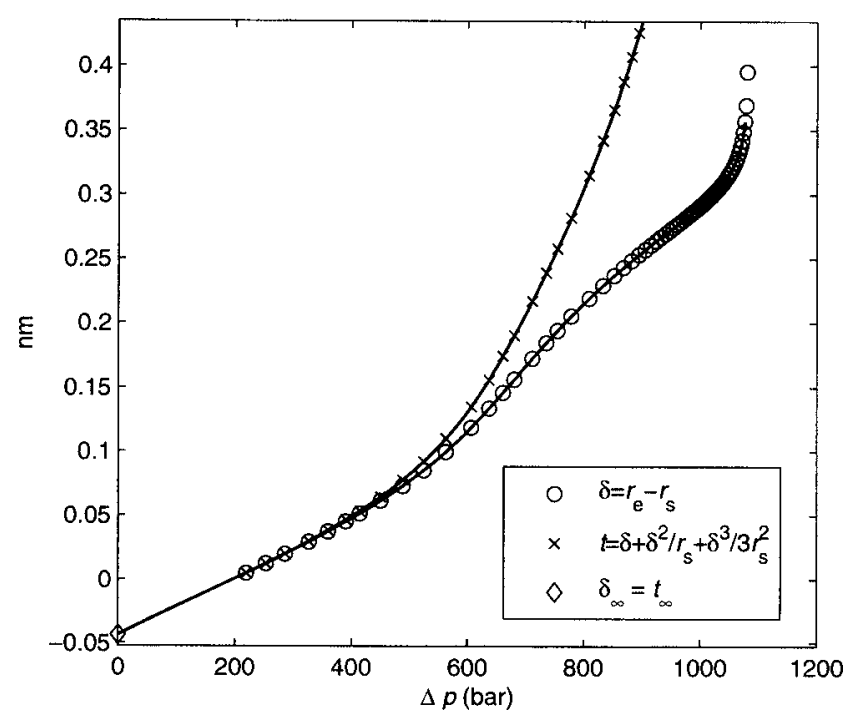

FIG. 5. Tolman length $\delta$ of $n$-nonane clusters at $230 \mathrm{~K}$ as a function of the pressure difference $\Delta p$. The solid line is based on Eq. (33).

requiring the relative difference to be smaller than $1 \mathrm{ppm}$ $\left(1 \times 10^{-6}\right)$. Also shown in Fig. 4 is the surface tension for the planar phase interface (diamond at $\Delta p=0$ ) computed with Eq. (23). With increasing $\Delta p$ the surface tension initially slightly increases, reaches a maximum, and then decreases along a line approximately according to a quarter of a circle, finally vanishing at the spinodal.

Figure 5 shows the Tolman lengths (circles) computed with Eqs. (9) and (30). The x symbols are the lengths $t$ computed using Eq. (14). Also shown is the Tolman length for the planar phase interface (diamond), computed using Eqs. (30)-(32).

The solid line in Fig. 4 is obtained by a piecewise cubic interpolation between these points. This line is a piecewise cubic,

$$
f_{i}(\Delta p)=h_{0 i}+h_{1 i} \Delta p+h_{2 i}(\Delta p)^{2}+h_{3 i}(\Delta p)^{3},
$$

with coefficients $h_{0 i}, \ldots, h_{3 i}$ obtained from the surface tensions and lengths $t$ in the boundary points, using Eq. (11),

$$
\begin{aligned}
& f_{i}\left(\Delta p_{i}\right)=\sigma_{i}, \quad f_{i}^{\prime}\left(\Delta p_{i}\right)=-t_{i}, \\
& f_{i}\left(\Delta p_{i+1}\right)=\sigma_{i+1}, \quad f_{i}^{\prime}\left(\Delta p_{i+1}\right)=-t_{i+1} .
\end{aligned}
$$

For the left conditions of the first interval we use the surface tension $\sigma_{\infty}$ and Tolman length $\delta_{\infty}$ for the plane phase interface computed using Eqs. (23) and (30), respectively. A great advantage of Eq. (34) is that it enables an accurate interpolation between the planar interface and the first computed cluster. Another advantage is in checking the numerical consistency of the computations. The values (surface tensions) are computed from the works of formation $\Delta \Omega$ solely, whereas the slopes are computed based on the excess numbers of molecules $\Delta N$. If the computed density profiles were inaccurate, the resulting line would be wavy. The solid line $t(\Delta p)$ interconnecting the $t$ values (crosses) shown in Fig. 5 is a piecewise quadratic curve obtained by derivation of the cubic Eq. (33), $t(\Delta p)=-(\mathrm{d} f / \mathrm{d} \Delta p)$. Here again we observe that the line is fluent. Numerical inconsistency would result

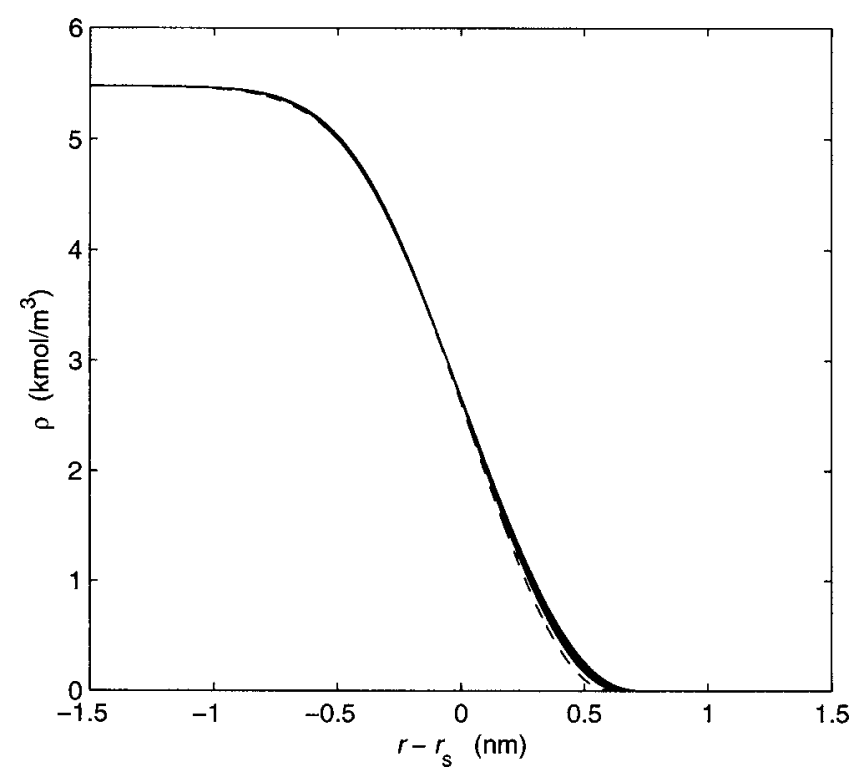

FIG. 6. Scaled density profiles of six largest clusters (solid lines) and the profile of the planar interface (dashed line). The profiles are translated such that the surfaces of tension of all profiles coincide. The scaled and translated density profiles are given as $\left[\rho\left(r-r_{s}\right)-\rho_{G}\right] \times\left(\Delta \rho_{\infty} / \Delta \rho\right)+\rho_{G_{\infty}}$.

into changes of slope at computed points (the crosses). Using the computed dependency $t(\Delta p)$, the solid line interconnecting the Tolman lengths (circles) is obtained by solving Eq. (14). The solid line $\Delta \Omega(\Delta p)$ showing the dependence of the work of formation on the pressure difference in Fig. 2 is obtained by substituting the interpolated surface tension Eq. (33) into Eq. (5) and solving it for $\Delta \Omega$. The solid line $\Delta N(\Delta p)$ in Fig. 3 is obtained by solving Eq. (13) for $\Delta N$ and using the previously computed $t(\Delta p)$ and the radius of tension $r_{s}$ obtained by substituting the interpolated surface tension Eq. (33) into the Laplace equation [Eq. (1)].

As shown in Fig. 5, the lengths $\delta$ and $t$ are initially practically identical and are represented by an almost linear function of $\Delta p$. Unlike the original result by Tolman, ${ }^{10}$ the Tolman length is initially negative, corresponding to an increase in surface tension. Both lengths turn positive at $\Delta p$ $\approx 200$ bars, where the surface tension has its maximum. For smaller clusters (large $\Delta p$ ) the length $t$ diverges faster from the straight line. On the other hand, the behavior of the Tolman length $\delta$ is more complex, showing two inversion points. The observed behavior of the Tolman length is in very good agreement with results by Gránásy ${ }^{31}$ obtained with the Van der Waals equation of state.

\section{DEPENDENCE OF THE SURFACE TENSION OF CRITICAL CLUSTERS ON THE PRESSURE DIFFERENCE $\Delta p$}

\section{A. A cluster model based on the density profile of the planar phase interface}

In Fig. 1, one observes that the density profiles of large clusters appear as copies of a horizontally translated curve. Figure 6 shows profiles of six largest clusters and the profile of the planar interface translated such that the surfaces of tension of all profiles coincide. It can be seen that the profiles almost collapse into one single curve. The only noticeable 
difference is the low-density tail which builds up on the gaseous side of the profile as the gas pressure is increased. This feature will be discussed later in greater detail. Besides the fact that the profiles almost coincide, we emphasize the circumstance that this happens when the surfaces of tension are taken as the origin of coordinates. A substantially worse result would be obtained when the equimolar surfaces were translated to the origin. This indicates that the position of the surface of tension with respect to the profile is fixed, whereas the position of the equimolar surface changes when the curvature is varied.

Inspired by these observations, we develop an approximate cluster model. Quantities related to the model will be denoted with a subscript $m$. We construct the density profile of a cluster of given radius of tension $r_{s m}$ in the following manner. Let $\rho_{\infty}(z),-\infty<z<\infty$, be a density profile of the planar phase interface with the origin positioned in the surface of tension $\left(z_{s}=0\right)$. The function $\rho_{\infty}\left(r-r_{s m}\right)$ is the profile translated in a manner that the original surface of tension is located at a radius $r_{s m}$. Further we create a mirror image $\rho_{\infty}\left(-r-r_{s m}\right)$, sum up both functions, and scale them linearly to a function

$$
y(r) \equiv \frac{\rho_{\infty}\left(r-r_{s m}\right)+\rho_{\infty}\left(-r-r_{s m}\right)-\rho_{L_{\infty}}-\rho_{G^{\infty}}}{\Delta \rho_{\infty}} .
$$

Here we denoted $\Delta \rho_{\infty} \equiv \rho_{L^{\infty}}-\rho_{G^{\infty}}$. Function $y(r)$ varies between zero (gas) and unity (liquid). The approximate density profile of a cluster is then obtained as

$$
\rho_{m}(r)=\rho_{G}+\Delta \rho y(r)
$$

where $\Delta \rho$ is the difference of densities of the bulk liquid and gas phases. The density difference $\Delta \rho$ depends on $r_{s m}$, but for now we leave it undetermined. By design, Eq. (36) provides an excellent approximation for relatively large clusters (droplets); for small clusters it is at least qualitatively correct - the derivative of the density with respect to $r$ vanishes at the center.

As a next step we express the excess number of molecules scaled by the density difference,

$$
\Delta N_{m} / \Delta \rho=\frac{1}{\Delta \rho} \int_{0}^{\infty}\left[\rho_{m}(r)-\rho_{G}\right] 4 \pi r^{2} \mathrm{~d} r=\int_{0}^{\infty} y(r) 4 \pi r^{2} \mathrm{~d} r
$$

Equation (37) can already be evaluated numerically. We show the dependence of $\Delta N_{m} / \Delta \rho$ on the radius of the surface of tension, $r_{s m}$. We substitute $r=r_{s m}+z$ and by a tedious derivation we obtain a simple result,

$$
\begin{aligned}
\Delta N_{m} / \Delta \rho & =\int_{-r_{s m}}^{\infty} y\left(r_{s m}+z\right) 4 \pi\left(r_{s m}+z\right)^{2} \mathrm{~d} z \\
& =4 \pi\left(\frac{1}{3} r_{s m}^{3}+K_{0} r_{s m}^{2}+2 K_{1} r_{s m}+K_{2}\right),
\end{aligned}
$$

where we introduced moments

$$
\begin{aligned}
K_{k} \equiv & \frac{1}{\Delta \rho_{\infty}}\left\{\int_{-\infty}^{0}\left[\rho_{\infty}(z)-\rho_{L^{\infty}}\right] z^{k} \mathrm{~d} z\right. \\
& \left.+\int_{0}^{\infty}\left[\rho_{\infty}(z)-\rho_{G^{\infty}}\right] z^{k} \mathrm{~d} z\right\} .
\end{aligned}
$$

We note that $K_{0}=\delta_{\infty}$. For a "symmetric" profile-profile whose derivative $\mathrm{d} \rho / \mathrm{d} z$ is an even function of $z$-all even moments $\left(K_{0}, K_{2}, \ldots\right)$ vanish. In this sense, $\delta_{\infty}$ is the most significant measure of the asymmetry. Further, we note that all odd moments $\left(K_{1}, K_{3}, \ldots\right)$ are necessarily positive. Let us consider a simplistic symmetric profile, linearly decreasing from $\rho_{L^{\infty}}$ at $z=-L / 2$ to $\rho_{G^{\infty}}$ at $z=L / 2$. The moments of this profile are $K_{0}=0, K_{1}=L^{2} / 24, K_{2}=0, K_{3}=L^{4} / 320, \ldots$. We see that $\left(K_{1}\right)^{1 / 2}$ characterizes the thickness of the profile, therefore, we introduce a thickness $L_{1}$, defined as

$$
L_{1} \equiv\left(24 K_{1}\right)^{1 / 2} \text {. }
$$

Having determined the moments $K_{0}, K_{1}$, and $K_{2}$, we continue the analysis of the surface tension of clusters. Substituting Eq. (38) for $\Delta N$ in Eq. (13) we obtain the length $t_{m}$,

$$
t_{m}=\frac{1}{A_{s m}}\left(\frac{\Delta N_{m}}{\Delta \rho}-V_{s m}\right)=K_{0}+2 K_{1} q_{s m}+K_{2} q_{s m}^{2},
$$

where $A_{s m} \equiv 4 \pi r_{s m}^{2}, V_{s m} \equiv \frac{4}{3} \pi r_{s m}^{3}$, and $q_{s m} \equiv 1 / r_{s m}$ is the curvature of the surface of tension. According to Eq. (11), the length $t$ determines the dependence of the surface tension on the pressure difference $\Delta p$. However, $t$ as given by Eq. (41) is a function of the curvature $q$, not of $\Delta p$. To obtain an integrable equation, we combine Eq. (11) with the Laplace equation Eq. (1) to

$$
\frac{\mathrm{d} \ln \sigma}{\mathrm{d} q}=-\frac{2 t(q)}{1+2 t(q) q} .
$$

We substitute Eq. (41) for $t$ and integrate,

$$
\frac{\sigma_{m}}{\sigma_{\infty}}=\exp \left[-2 \int_{0}^{q_{s m}} \frac{K_{0}+2 K_{1} q+K_{2} q^{2}}{1+2 K_{0} q+4 K_{1} q^{2}+2 K_{2} q^{3}} \mathrm{~d} q\right] .
$$

The integral can be easily evaluated by numerical quadrature. Once the surface tension is given as a function of $q_{s m}$, we compute the corresponding pressure difference from the Laplace equation $\Delta p=2 \sigma_{m} q_{s m}$. The corresponding pressure of the gas phase is obtained by solving the equation $\mu_{L}\left(p_{G}\right.$ $+\Delta p)=\mu_{G}\left(p_{G}\right)$. Liquid and gas densities are obtained from the equation of state for the respective pressures.

For moderate $\Delta p$ it is possible to develop a Taylor expansion of surface tension with respect to $\Delta p$ by combining Eq. (43) and the Laplace equation. In this way we find a series

$$
\sigma_{m}=\sigma_{\infty}-K_{0} \Delta p-\frac{K_{1}}{2 \sigma_{\infty}} \Delta p^{2}-\frac{4 K_{0} K_{1}+K_{2}}{12 \sigma_{\infty}^{2}} \Delta p^{3}+\ldots
$$

The cubic and higher terms become significant at high $\Delta p$, where the center of the cluster is influenced by the phase interface and, consequently, the original function Eq. (43) is 


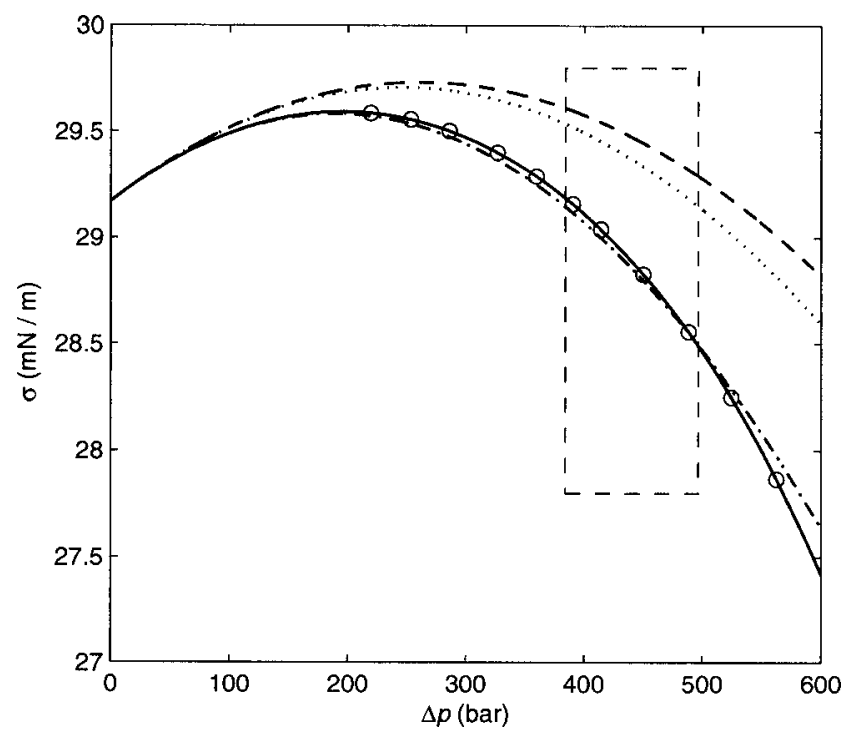

FIG. 7. (Circles) Surface tension computed with the gradient theory. (Solid line) Interpolating function Eq. (34). (Dashed line) Surface tension given by Eq. (43). (Dotted line) Approximation by Eq. (45). (Dash-dotted line) quadratic expansion Eq. (47). The rectangle shows the range of critical clusters relevant for nucleation experiments (Refs. 17, 22, and 24).

inaccurate. Therefore, we reduce our analysis to the quadratic representation of the surface tension,

$$
\sigma_{m} \approx \sigma_{\infty}-\delta_{\infty} \Delta p-\frac{L_{1}^{2}}{48 \sigma_{\infty}} \Delta p^{2},
$$

and the corresponding linear expression for the length $t$,

$$
t_{m} \approx \delta_{\infty}+\frac{L_{1}^{2}}{24 \sigma_{\infty}} \Delta p
$$

We determined that $\delta_{\infty}<0$. Therefore, the function $\sigma_{m}(\Delta p)$ is initially increasing in exact agreement with the density gradient results. The quadratic term is positive, so that the function $\sigma_{m}(\Delta p)$ bends down, forming a maximum. However, the magnitude of the coefficient of the quadratic term is insufficient to fully explain the shape of the $\sigma_{m}(\Delta p)$ curve as computed by the gradient theory.

Figure 7 shows that the quadratic expansion, Eq. (45), is a good approximation to the values obtained from the original approximation given by Eq. (43). Both equations overestimate the surface tension as given by the gradient theory. Also shown is a quadratic expansion of the gradient theory results,

$$
\sigma \approx \sigma_{\infty}+(\mathrm{d} \sigma / \mathrm{d} \Delta p)_{\infty} \Delta p+\frac{1}{2}\left(\mathrm{~d}^{2} \sigma / \mathrm{d} \Delta p^{2}\right)_{\infty}(\Delta p)^{2} .
$$

The last equation provides a good approximation to the gradient theory results in a broad range of $\Delta p$, including the range relevant for nucleation experiments. The linear terms of Eqs. (45) and (47) are equal. The only difference is in the quadratic term. The approximative quadratic coefficient $-L_{1}^{2} /\left(48 \sigma_{\infty}\right)$ represents a major part $(77 \%$, in the case computed) of the accurate coefficient $\frac{1}{2}\left(\mathrm{~d}^{2} \sigma / \mathrm{d} \Delta p^{2}\right)_{\infty}$.

\section{B. Effect of the low-density tail of the density profile}

A subtle feature can be recognized in Fig. 6: Abuildup of the low-density tail of the density profile. We will show that

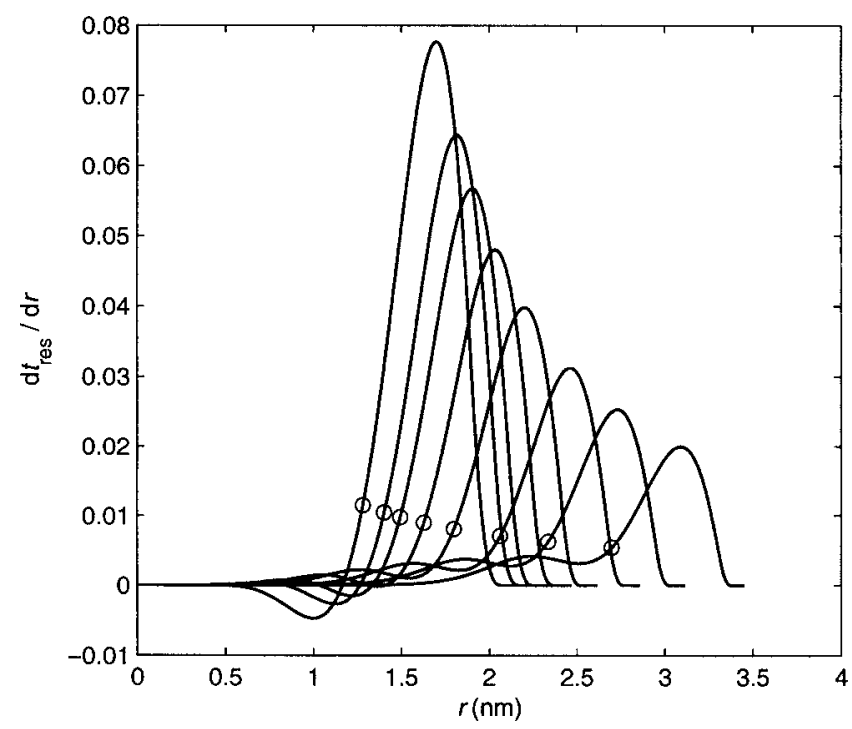

FIG. 8. Radial distribution of the residual $t_{\text {res }}$ approximated by Eq. (50) for eight largest clusters. The curves are obtained for individual clusters as $d t_{\text {res }} / d r \approx\left[\rho(r)-\rho_{m}\left(r_{s} ; r\right)\right] r^{2} /\left(\Delta \rho r_{s}^{2}\right)$, where $\rho(r)$ is the profile given by the gradient theory, and $\rho_{m}\left(r_{s} ; r\right)$ is the cluster model based on the density profile of the planar phase interface. The circles indicate the position of the surface of tension $\left(r_{s}\right)$. The smaller the cluster, the higher the maximum.

this feature is responsible for the the difference between the gradient theory results and the predictions based on the cluster model Eq. (36). We analyze the difference in terms of the length $t$. We define a residual

$$
t_{\text {res }} \equiv t-t_{m}
$$

where $t$ is given by the full gradient theory and $t_{m}$ is given by Eq. (41). Both $t$ and $t_{m}$ are evaluated for the same thermodynamic condition of the gas phase, that means for the same $\Delta p$. By iterating Eq. (43) and the Laplace equation for the given $\Delta p$ we find a radius of tension, $r_{s m}$. Because $t_{m}$ in Eq. (48) corresponds to the approximate radius $r_{s m}$, we denote it as $t_{m}\left(r_{s m}\right)$. The value of $r_{s m}$ slightly differs from the accurate radius $r_{s}$, obtained directly from the gradient theory via Eq. (4). To be able to elucidate the origin of $t_{\text {res }}$, we also construct the model cluster, Eq. (36), for the exact $r_{s}$ and compute the corresponding $t_{m}\left(r_{s}\right)$. We modify Eq. (48) to

$$
t_{\text {res }}=\left[t-t_{m}\left(r_{s}\right)\right]+\left[t_{m}\left(r_{s}\right)-t_{m}\left(r_{s m}\right)\right] .
$$

We will show later that the expression in the second square brackets is negligible. The residual $t_{\text {res }}$ is essentially given by the first bracketed expression. Using Eqs. (13) and (29) we find

$$
t_{\mathrm{res}} \approx t-t_{m}\left(r_{s}\right)=\int_{0}^{\infty} \frac{\left[\rho(r)-\rho_{m}\left(r_{s} ; r\right)\right] r^{2}}{\Delta \rho r_{s}^{2}} \mathrm{~d} r,
$$

where $\rho(r)$ is the density profile of the cluster as given by the gradient theory and $\rho\left(r_{s} ; r\right)$ is the density profile of the model cluster Eq. (36) constructed for given accurate $r_{s}$. Figure 8 shows the integrands of Eq. (50) for eight largest clusters as functions of the radial coordinate $r$. The circle on each curve indicates the position of the surface of tension $\left(r_{s}\right)$. Most significant feature is the hump right of the surface of tension, corresponding to the low-density tail of the density profile. The residual $t_{\text {res }}$ is essentially given by the area below these 
humps. With increasing $\Delta p$ (and correspondingly decreasing $r_{s}$ ), the width of the humps remains about the same, but the height increases proportionally to $\Delta p$. This gives rise to the proportionality of $t_{\text {res }}$ to $\Delta p$.

We assumed that the expression in the second square brackets in Eq. (49) can be neglected. We prove this assumption. For $t_{m}\left(r_{s m}\right)$ we use expression Eq. (41). The same expression, but with $q_{s m}$ exchanged for $q_{s} \equiv 1 / r_{s}$, can be used for $t_{m}\left(r_{s m}\right)$. In this way we obtain

$$
t_{m}\left(r_{s}\right)-t_{m}\left(r_{s m}\right)=2 K_{1}\left(q_{s}-q_{s m}\right)+K_{2}\left(q_{s}^{2}-q_{s m}^{2}\right) .
$$

Substituting further from the corresponding forms of the Laplace equation $q_{s}=\Delta p /(2 \sigma)$, and $q_{s m}=\Delta p /\left(2 \sigma_{m}\right)$, using expansions Eq. (44) for $\sigma_{m}(\Delta p)$, and Eq. (47) for $\sigma(\Delta p)$, and performing Taylor expansion, we obtain

$$
\begin{aligned}
t_{m}\left(r_{s}\right)-t_{m}\left(r_{s m}\right)= & -\frac{K_{1}}{2 \sigma_{\infty}^{2}}\left[\frac{K_{1}}{\sigma_{\infty}}+\left(\frac{\mathrm{d}^{2} \sigma}{\mathrm{d} \Delta p^{2}}\right)_{\infty}\right](\Delta p)^{3} \\
& +o\left[(\Delta p)^{4}\right] .
\end{aligned}
$$

We conclude that approximation Eq. (50) is accurate except for terms of the order of $(\Delta p)^{3}$ and higher, which become significant at rather high $\Delta p$. This justifies the use of Eq. (50) to explain the origin of the residual $t_{\text {res }}$.

\section{VAPOR-LIQUID NUCLEATION OF $n$-NONANE}

\section{A. Noncritical clusters and nucleation kinetics}

The gradient theory (the density functional theory in general) in the present form only enables computation of critical clusters. However, the works of formation for noncritical clusters can be estimated. The relatively easy case that the vapor is an ideal gas was studied by Talanquer and Oxtoby. ${ }^{40}$ Interaction with the gas phase can be incorporated applying the "noncritical nucleation theorem," derived by Hruby $^{41}$ and Bowles et al. ${ }^{42}$ We assume that the cluster is defined by some microscopic constraint. For the case of clusters in the gas phase, a suitable constraint is the number of molecules $N$ forming a cluster. To fix ideas, the Band $^{43}$ -Stillinger ${ }^{44}$ cluster of overlapping spheres can be assumed. When the condition of the vapor changes at constant temperature and constant $N$, the theorem says that the work of formation is changed as

$$
\mathrm{d}\left(\Delta \Omega_{N}\right)=-\Delta N_{N} \mathrm{~d} \mu_{G} .
$$

From this relation, the classical nucleation theorem ${ }^{45}$ can be derived. Here another aspect is important. By integrating at constant $N$, changing the condition of the vapor isothermally from the chemical potential $\mu_{N}^{*}$, at which the cluster of $N$ molecules is critical, up to the actual chemical potential of the gas phase $\mu_{G}$, we obtain

$$
\Delta \Omega_{N}\left(\mu_{G}\right)=\Delta \Omega_{N}\left(\mu_{N}^{*}\right)-\int_{\mu_{N}^{*}}^{\mu_{G}} \Delta N_{N} \mathrm{~d} \mu .
$$

Here, $\Delta \Omega_{N}\left(\mu_{N}^{*}\right)$ is the work of formation of the cluster of $N$ molecules computed with the gradient theory, and $\Delta \Omega_{N}\left(\mu_{G}\right)$ is the work of formation of the noncritical cluster. It is always possible to express the excess number of molecules as

$$
\Delta N_{N}=N-V_{N} \rho_{G},
$$

where $V_{N}$ represents the volume of the cluster of $N$ molecules. Unfortunately, $N$ is not defined in the gradient theory. A rational option is to take the volume of the sphere of tension for the volume of the cluster, $V_{N}=V_{s}=\frac{4}{3} \pi r_{s}^{3}$, and to obtain $N$ from Eq. (55). We proceed with integrating Eq. (54):

$$
\Delta \Omega_{N}\left(\mu_{G}\right)=\Delta \Omega_{N}\left(\mu_{N}^{*}\right)-N \cdot\left(\mu_{G}-\mu_{N}^{*}\right)+\int_{\mu_{N}^{*}}^{\mu_{G}} V_{N} \rho_{G} \mathrm{~d} \mu .
$$

Making an approximation that $V_{N}$ is independent of the condition of the gas, we find

$$
\Delta \Omega_{N}\left(\mu_{G}\right)=\Delta \Omega_{N}\left(\mu_{N}^{*}\right)-N \cdot\left(\mu_{G}-\mu_{N}^{*}\right)+V_{N} \cdot\left(p_{G}-p_{N}^{*}\right),
$$

where $p_{N}^{*}$ is the pressure of the gas phase at the condition when the cluster of $N$ molecules is critical. In comparison with treatment of Talanquer and Oxtoby, ${ }^{40}$ Eq. (57) does not neglect the interactions of the cluster with surrounding gas phase.

The number density of noncritical clusters of $N$ molecules is then obtained as

$$
n_{N}=\frac{1}{\vartheta} \exp \left[-\frac{\Delta \Omega_{N}\left(\mu_{G}\right)}{k_{B} T}\right] .
$$

In this equation, the prefactor $1 / \vartheta$ accounts for the translational degrees of freedom of the cluster. The prefactor is subject to ongoing discussions. ${ }^{35,46-48}$ For the present computations we used the classical ${ }^{1}$ choice of $1 / \vartheta=n_{1} \approx \rho_{G}$. The next standard step is determining the impingement rate $C_{N}$ of vapor molecules on the surface of cluster of $N$ molecules,

$$
C_{N}=A_{N} n_{1} \sqrt{\frac{k_{B} T}{2 \pi M}} .
$$

Here $A_{N}$ is the surface area of the cluster, approximated as $4 \pi r_{s}^{2}, n_{1}$ is number density of free vapor molecules (monomers), and $M$ is molecular mass of the vapor. By solving the nucleation kinetics in the steady state we obtain the nucleation rate

$$
J=\left(\sum_{N=N_{\min }}^{N_{\max }} \frac{1}{C_{N} n_{N}}\right)^{-1} .
$$

Theoretically, the summation should run from 1 to $\infty$. The actual minimum and maximum cluster sizes were taken as $N_{\min }=2$ and $N_{\max }=800$. The computed nucleation rates were insensitive to variation of these bounds, because the terms of Eq. (60), corresponding to clusters much smaller or bigger than the critical cluster, are small.

\section{B. Comparison with experimental nucleation rates}

The nucleation rate of $n$-nonane in helium has been measured by Hung et al. ${ }^{24,25}\left(233-313 \mathrm{~K}, 5 \times 10^{-5}-1 \mathrm{~cm}^{-3} \mathrm{~s}^{-1}\right)$ and by Rudek et al..$^{27}\left(257-313 \mathrm{~K}, 5 \times 10^{-4}-5 \mathrm{~cm}^{-3} \mathrm{~s}^{-1}\right)$ using an upward diffusion cloud chamber. The two data sets are consistent. Measurements by Rudek et al. are not considered 
here because they are at higher temperatures. Expansion chamber measurements have been reported by Adams et al. ${ }^{20}$ Wagner and Strey, ${ }^{21}$ and Viisanen et al. ${ }^{22}$ (in argon at $230 \mathrm{~K}, \approx 0.5$ bar, $\left.4 \times 10^{5}-2 \times 10^{8} \mathrm{~cm}^{-3} \mathrm{~s}^{-1}\right)$. Of the expansion chamber measurements, only the data by Viisanen $e t$ al. have been considered, because of their superior quality. Expansion wave tube experiments have been reported by Luijten et $a l .{ }^{17,28}$ The data by Luijten $e t$ al. are at higher pressures: Nominally 10, 25, and 40 bars. Only the 10 bars measurements were used for the present comparisons.

For the expansion chamber and expansion wave tube experiments we reevaluated the experimental supersaturations starting from the experimental temperatures, pressures, and molar fractions. We used the saturated vapor pressure equation as given by King and Al-Najjar, ${ }^{49}$ which was utilized by Hung $e t$ al. $^{24}$ to evaluate their diffusion cloud chamber data. We also considered the real-gas effects on supersaturation. The supersaturations were determined using definition

$$
S=\exp \left(\frac{\mu_{v}-\mu_{v, \text { sat }}}{k_{B} T}\right)
$$

where $\mu_{v}$ is the chemical potential of the vapor ( $n$-nonane) in the nucleation condition and $\mu_{v \text {,sat }}$ is the chemical potential of the vapor at phase equilibrium with liquid, in the presence of the background gas ( $\mathrm{He}$ or $\mathrm{Ar}$ ) at the same chemical potential $\mu_{g}$ as in the nucleation condition. For the gas phase we used the virial equation of state truncated after the second virial coefficient. For the argon-argon virial coefficient we used the correlation by Bokis and Donohue ${ }^{50}$ The argonnonane virial coefficient was computed according to Kaul and Prausnitz. ${ }^{51}$ The second virial coefficients for heliumhelium and helium-nonane were neglected. The supersaturation [Eq. (61)] also contains the pointing effect due to the pressure of the background gas. As discussed by Fisk and Katz, ${ }^{52}$ the pointing effect influences both the experimental supersaturations and the nucleation theory in a very similar fashion. To enable a direct comparison of nucleation data obtained at different background pressures, we defined a supersaturation reduced to pure vapor condition as

$$
S_{0}=S \exp \left(\frac{p-p_{v, \text { sat }}}{k_{B} T \rho_{L}}\right)
$$

The experimental data are at temperatures slightly deviating (up to $4 \mathrm{~K}$ ) from the $T_{\text {nom }}=230 \mathrm{~K}$ isotherm. We adjusted the supersaturations further to compensate for this,

$$
S_{\text {corr }}=S_{0} \frac{S_{\text {ICCT }}\left(J_{\text {exper }}, T_{\text {nom }}\right)}{S_{\text {ICCT }}\left(J_{\text {exper }}, T_{\text {exper }}\right)},
$$

where $S_{\text {ICCT }}(J, T)$ is the inverse function to the so-called internally consistent classical nucleation theory as given by Girshick and Chiu. ${ }^{46}$ The experimental nucleation rate data shown in Fig. 9 are corrected according to Eq. (63).

Figure 9 also shows the theoretical line computed with the gradient theory. This line was computed for given supersaturations using Eq. (60). Also shown is the classical nucleation theory by Becker and Döring

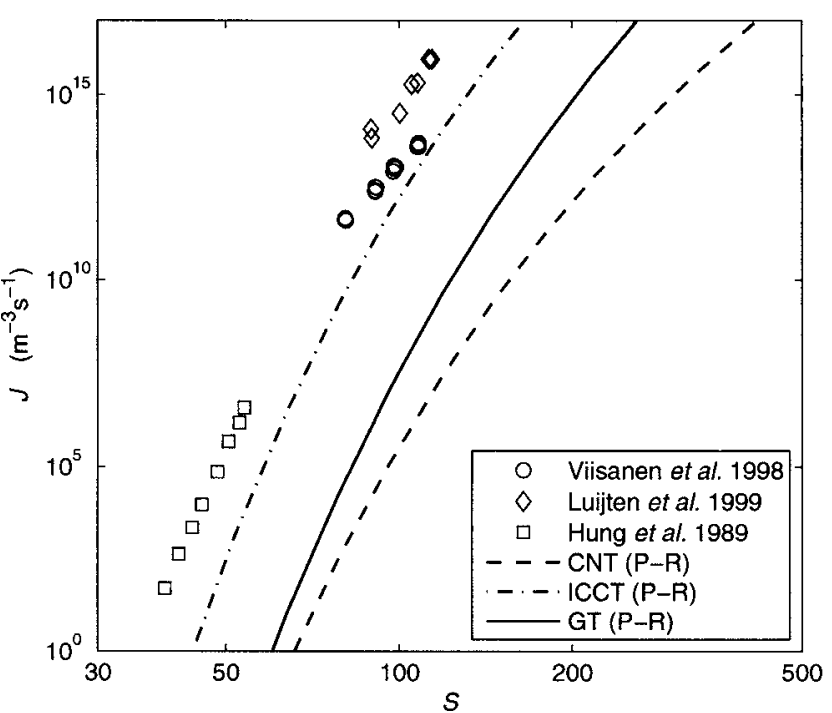

FIG. 9. Nucleation rate $J$ of $n$-nonane clusters at $230 \mathrm{~K}$ as function of supersaturation $S$. (Circles, squares, and diamonds) Nucleation rate data by Viisanen et al. (Ref. 22), Luijten et al. (Refs. 17 and 28), and Hung et al. (Refs. 24 and 25), respectively. Following theoretical predictions are computed with the Peng-Robinson equation of state. (Dashed line) Classical nucleation theory (CNT) by Becker and Döring (Ref. 1), (dash-doted line) internally consistent classical theory (ICCT) by Girshick and Chiu (Ref. 46), and (solid line) gradient theory (GT) (this work).

$$
J_{\mathrm{CNT}}=\frac{\rho_{G}^{2}}{\rho_{L_{\infty}}}\left(\frac{2 \sigma_{\infty}}{\pi M}\right)^{1 / 2} \exp \left[-\frac{4 \theta^{3}}{27(\ln S)^{2}}\right],
$$

and the internally consistent theory by Girshick and Chiu

$$
J_{\mathrm{ICCT}}=\frac{\rho_{G} \rho_{G^{\infty}}}{\rho_{L^{\infty}}}\left(\frac{2 \sigma_{\infty}}{\pi M}\right)^{1 / 2} \exp \left[\theta-\frac{4 \theta^{3}}{27(\ln S)^{2}}\right],
$$

where $M$ is the molecular mass and $\theta$ is the dimensionless surface tension,

$$
\theta \equiv \sigma_{\infty}(36 \pi)^{1 / 3} \rho_{L^{\infty}}^{-2 / 3} / k_{B} T .
$$

We also computed nucleation rates using the classical formula Eq. (64) in which the exponential $\exp \left[-4 \theta^{3} / 27(\ln S)^{2}\right]$ was replaced with $\exp \left(-\Delta \Omega_{\text {crit }} / k_{B} T\right)$, where $\Delta \Omega_{\text {crit }}$ was the work of formation of the critical cluster computed with the gradient theory. The resulting nucleation rates were about $10 \%$ higher than the nucleation rates computed with the detailed kinetics approach given in Sec. V A.

The gradient theory computations are done with the Peng-Robinson equation of state, which yields rather inaccurate vapor pressure and liquid density for $n$-nonane at $230 \mathrm{~K}$. To enable a comparison of the theories, we computed the classical and internally consistent theories with the liquid density and vapor pressure given by the Peng-Robinson equation of state. The comparison with the classical theory is then quite clear: At low supersaturations the gradient theory gives lower nucleation rates than the classical theory. This is due to the fact that, for the large critical clusters, the surface tension obtained with the gradient theory (Fig. 4) is slightly higher than the planar surface tension used in the classical theory. For higher supersaturations, the critical and nearcritical clusters playing the decisive role in the nucleation kinetics get smaller. According to Fig. 4, the corresponding surface tension gets smaller than at the planar phase interface 


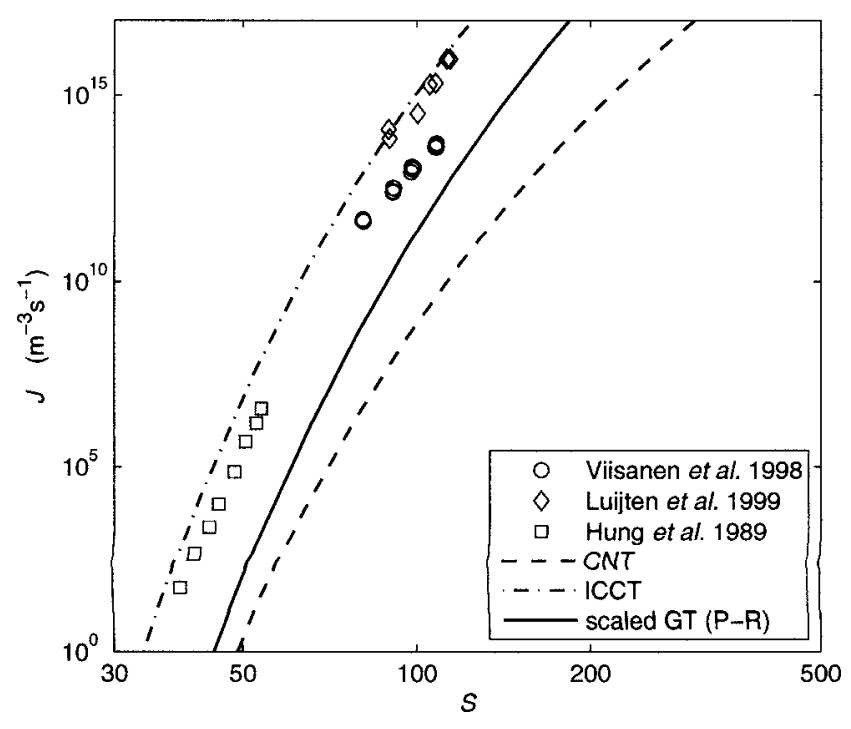

FIG. 10. Same as Fig. 9, except that the theoretical predictions CNT and ICCT are computed using accurate saturated liquid density and vapor pressure. The the gradient theory results are scaled based on the CNT (see the text).

and the nucleation rate predicted by the gradient theory gets higher than for the classical nucleation theory. As a result, the gradient theory computations predict a steeper $\log J$ versus $\log S$ line. The internally consistent theory predicts nucleation rates much closer to the experimental ones. This is mainly due to a different prefactor $1 / \vartheta$ in formula (58), which is subject of ongoing discussions. In this paper we do not want to enter this discussion and we use the prefactor corresponding to the original Becker-Döring theory. In general this prefactor, whatever its value might be, is rather insensitive to supersaturation. It means that the ratio of experimental and theoretical nucleation rates should be almost constant in a broad range of supersaturations. In this respect the gradient theory performs better than the classical nucleation theory.

As already mentioned, the performance of the PengRobinson equation of state at $230 \mathrm{~K}$ is poor. Because we wanted to maintain the compatibility of the present results with a parallel work on gradient theory in hydrocarbon mixtures, we did not proceed, at this stage, by adjusting the parameters of the Peng-Robinson equation of state. In Fig. 10 we show the nucleation rates predicted with the classical nucleation theory and the internally consistent classical nucleation theory, evaluated for accurate values of saturated liquid density ${ }^{53}$ and vapor pressure. ${ }^{49}$ Now the ICCT slightly overestimates and the CNT is substantially closer than for the Peng-Robinson values. Because the gradient theory was developed here as a modification of the classical nucleation theory with a specific dependency of the surface tension on $\Delta p$, it is possible to estimate the focus of the gradient theory line corrected for the accurate saturated liquid density and saturated vapor pressure: We denote the ratio of densities of saturated liquid as given by Peng-Robinson equation state to the accurate saturated liquid density as $\ell$ $\equiv\left(\rho_{L_{\infty}}\right)_{\mathrm{P}-\mathrm{R}} /\left(\rho_{L^{\infty}}\right)_{\text {accur }}$, and the ratio of densities of saturated vapor as given by Peng-Robinson equation state to the accurate saturated vapor density as $g \equiv\left(\rho_{G^{\infty}}\right)_{\mathrm{P}-\mathrm{R}} /\left(\rho_{G^{\infty}}\right)_{\text {accur }}$. The scaled supersaturation is given as $S_{\text {scaled }}=S^{\ell}$ and the scaled nucleation rate as $J_{\mathrm{GT}, \text { scaled }}=J_{\mathrm{GT}} \cdot\left(S_{\text {scaled }} / S\right)^{2} \cdot \ell / g^{2}$.

The ratio of the experimental data by Hung et al. ${ }^{24}$ and Luijten et al. ${ }^{17}$ to the scaled results of the gradient theory is practically constant in the whole range of supersaturation. The slope of the data by Viisanen et al. $^{22}$ agrees more with the classical nucleation theory.

\section{CONCLUSIONS}

We performed gradient theory computations of the density profiles, works of formation, and excess numbers of molecules of $n$-nonane clusters. These results were interpreted in the framework of the Gibbsian droplet model with variable surface tension. The difference of pressures $\Delta p$ of the liquid phase and vapor phase in chemical equilibrium was used as a variable characterizing the supersaturation. This interpretation allowed construction of an efficient interpolating scheme [Eq. (33)] and a check of numerical accuracy of the computations.

We devised a cluster model [Eqs. (35) and (36)] allowing us to predict the dependency of the surface tension on the pressure difference based on the density profile of the planar phase interface. The application of the model is quite simple: Using Eq. (22) we compute the density profile of the planar phase interface. Mathematically this is done by quadrature of an integral - a task much easier than solving the boundary value problem for the Euler-Lagrange equation [Eq. (24)]. The position of the surface of tension for the planar density profile is found using Eq. (32), and the origin of coordinates is shifted to the surface tension. Moments $K_{0}$ and $K_{1}$ of the density profile are obtained using Eq. (39). From these moments we obtain characteristic lengths of the profile: $\delta_{\infty}$ $=K_{0}$ characterizes the asymmetry of the density profile; $L_{1}$ $=\left(24 K_{1}\right)^{1 / 2}$ characterizes the thickness of the profile. Using these lengths it is possible to compute a quadratic approximation of the surface of tension, Eq. (45).

We demonstrated that the difference of the cluster surface tension as predicted by the cluster model from the gradient theory computations is due to the buildup of lowdensity tail of the density profile. The tail of the density profile on the gas-phase side grows with increasing supersaturation. This feature breaks the similarity of the density profiles assumed in the cluster model. The buildup of the low-density tail contributes a minor part $(23 \%$, in the case computed) to the quadratic term of the function $\sigma(\Delta p)$, but this contribution is important in the supersaturation range corresponding to nucleation experiments. As a consequence, the model significantly overestimates the surface tension in the range relevant to nucleation experiments (cf. Fig. 7). The model is not directly related to the gradient theory-it relies on general Eq. (11). This opens the possibility of using it to estimating the size dependency of the surface tension if the planar density profile and the corresponding surface of tension are determined with different simulation methods.

We also developed a way of estimating the works of formation of noncritical cluster in order that the complete nucleation kinetics can be evaluated. Finally, we computed the nucleation rate by adaptation of the standard steady state 
nucleation kinetics and compared the theoretical prediction to the experimental data. The prediction is not yet fully quantitative for several reasons. First, the performance of the Peng-Robinson equation of state at low temperatures (with respect to the critical point) is poor. Second, unresolved problem is the exact magnitude of the prefactor of Eq. (58), representing the translational degrees of freedom of the cluster. Upon the condition that a sufficiently accurate mean-field equation of state is available, the gradient theory is, with certainty, superior to the capillarity approximation, assuming a constant surface tension. On the other hand, it is to be realized that the gradient theory itself contains approximations. It is to be expected that the gradient theory performs well if the gradient length of the density profile is large in comparison with the range of the intermolecular forces. For the examples considered in this paper, this condition is only marginally met.

\section{ACKNOWLEDGMENTS}

The authors gratefully acknowledge the support by Grant No. ESF.6472 of the Technology Foundation STW, The Netherlands, and by Grant No. 101/05/2214 of the Grant Agency of the Czech Republic.

\section{APPENDIX A: SOME DERIVATIONS}

In this appendix we derive several results of the gradient theory important for the present paper. Some analogous equations found in the literature ${ }^{5}$ are based on the pressure tensor. In this paper we avoided the introduction of the pressure tensor.

We first derive the formulas Eq. (27) and Eq. (28) suitable for the computation of the work of formation $\Delta \Omega$ for a given density profile $\rho(r)$. We make use of the fact that $\mathrm{d} \Delta \omega_{\text {hom }} / \mathrm{d} r=\Delta \mu \cdot \mathrm{d} \rho / \mathrm{d} r$. Using the Euler-Lagrange equation [Eq. (24)], we obtain

$$
\frac{\mathrm{d} \Delta \omega_{\mathrm{hom}}}{\mathrm{d} r}=\frac{c}{2} \frac{\mathrm{d}}{\mathrm{d} r}\left(\frac{\mathrm{d} \rho}{\mathrm{d} r}\right)^{2}+\frac{2 c}{r}\left(\frac{\mathrm{d} \rho}{\mathrm{d} r}\right)^{2} .
$$

By integration from $r$ to $\infty$ and using the fact that $\Delta \omega_{\text {hom }}$ vanishes in the limit $r \rightarrow \infty$, we obtain

$$
\Delta \omega_{\mathrm{hom}}=\frac{c}{2}\left(\frac{\mathrm{d} \rho}{\mathrm{d} r}\right)^{2}-Q(r),
$$

where

$$
Q(r) \equiv \int_{r}^{\infty} \frac{2 c}{r^{\prime}}\left(\frac{\mathrm{d} \rho}{\mathrm{d} r^{\prime}}\right)^{2} \mathrm{~d} r^{\prime}
$$

From Eq. (A2) we see that the quantity $Q(r)$ as the following limits:

$$
Q(0)=-\Delta \omega_{\text {hom }}[\rho(0)], \quad Q(\infty)=0 .
$$

We also note the following identity obtained by integration by parts:

$$
\int_{0}^{\infty} Q(r) 4 \pi r^{2} \mathrm{~d} r=\frac{2}{3} \int_{0}^{\infty} c\left(\frac{\mathrm{d} \rho}{\mathrm{d} r}\right)^{2} 4 \pi r^{2} \mathrm{~d} r .
$$

Now we substitute $\Delta \omega$ given by Eq. (A2) into the original expression Eq. (17) for the work of formation. Using further Eq. (A5) we obtain immediately Eq. (27), where the integrand is the square gradient only. Further, by a linear combination of $3 \Delta \Omega$ given by Eq. (A5) and $-2 \Delta \Omega$ given by Eq. (17), we recover expression (28) for the work of formation, where the integrand is $\Delta \omega_{\text {hom }}$.

We utilize function $Q(r)$ once more to derive a formula for the computation of the radius of the surface of tension $r_{s}$, which will be particularly useful for proving Eq. (32) used to determine the position of the surface of tension for the planar phase interface. For a large cluster (a droplet) whose center is not affected by the phase interface, the density at $r=0$ is equal to the density of the bulk liquid phase $\rho_{L}$ corresponding to a given chemical potential $\mu_{L}=\mu_{G}$. Consequently, realizing that the grand potential density of a homogeneous system equals $-p$, the excess grand potential density reduces to the negative of the pressure difference,

$$
\Delta \omega_{\text {hom }}\left(\rho_{L}\right)=\left[\phi_{\text {hom }}\left(\rho_{L}\right)-\mu_{L} \rho_{L}\right]+p_{G}=-\Delta p .
$$

Because of the properties (A4) of function $Q(r)$, we have

$$
\Delta p=Q(0)=\int_{0}^{\infty} \frac{2 c}{r}\left(\frac{\mathrm{d} \rho}{\mathrm{d} r}\right)^{2} \mathrm{~d} r .
$$

We express the radius of the surface of tension, $r_{s}$, using Eq. (4) and use Eq. (27) for $\Delta \Omega$ and Eq. (A7) for $\Delta p$,

$$
r_{s}^{\prime 3}=\frac{\int_{0}^{\infty} c(\mathrm{~d} \rho / \mathrm{d} r)^{2} r^{2} \mathrm{~d} r}{\int_{0}^{\infty} c(\mathrm{~d} \rho / \mathrm{d} r)^{2} r^{-1} \mathrm{~d} r} .
$$

This expression gives an approximation $r_{s}^{\prime}$ of the radius of tension $r_{s}$. The approximation consists in the assumption that the density in the center reaches the bulk density $\rho_{L}$ corresponding to the given chemical potential. This assumption is certainly met for macroscopic droplets. The present results (cf. Fig. 11) indicate that the convergence of $r_{s}^{\prime} \rightarrow r_{s}$ as $r_{s}$ $\rightarrow \infty$ is extremely fast: Already when the radius is about the same as the thickness of the phase interface [represented with $L_{1}$, Eq. (40)], the relative difference is $1 \times 10^{-4}$; for a radius which is twice the thickness, the difference is smaller than $1 \times 10^{-7}$, reaching the numerical accuracy. The observed relative deviations $\left(r_{s}^{\prime} / r_{s}-1\right)$ can be approximated with a function $6 \times 10^{-4} \cdot\left(L_{1} / r_{s}^{\prime}\right)^{17.0}$. We note the extreme value of the exponent. For clusters whose center is significantly influenced by the phase interface, $r_{s}^{\prime}$ is a more rational definition of the radius; it stays in the neighborhood of the inflection point of the phase interface, whereas $r_{s}$ is too small. Figure 11 shows that $r_{s}^{\prime}$ has a minimum value of about $0.6 L_{1}$. This is a general feature of the mean-field theory close to the spinodal: ${ }^{39}$ The spatial extent of the cluster increases (diverges at the spinodal), whereas the amplitude (density difference) decreases. In the whole range, we observe $r_{s}^{\prime}>r_{s}$; this indicates that $\Delta p$ evaluated with Eq. (A7) is smaller than the exact value given by equality of chemical potentials Eq. (2). 


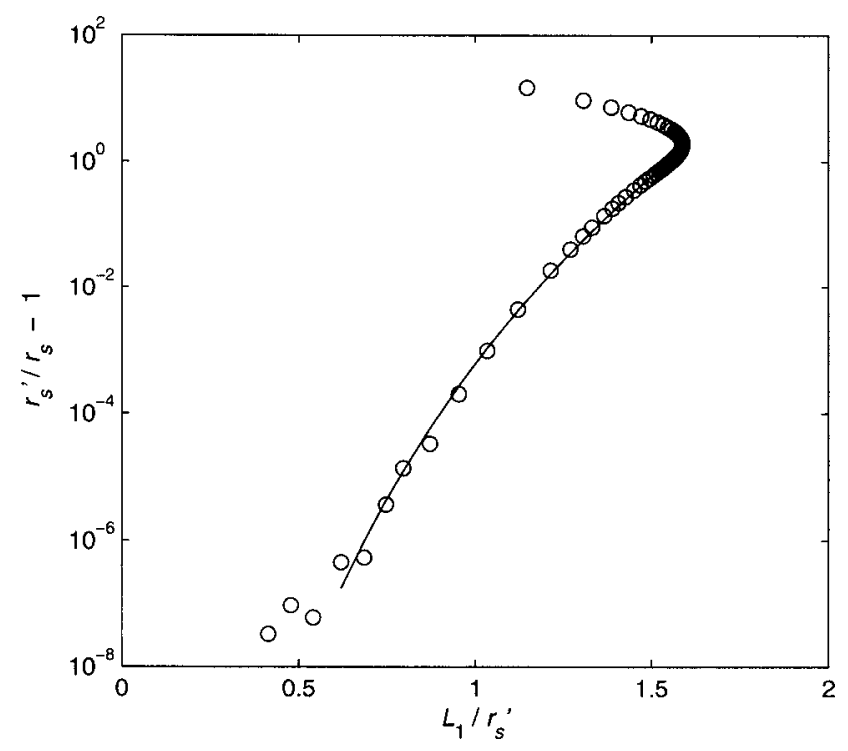

FIG. 11. Relative deviation of the radius of the surface tension $r_{s}^{\prime}$ computed with Eq. (A8) from $r_{s}$ determined with Eq. (4) as a function of the ratio of the width of the phase interface $L_{1}$ and the radius $r_{s}^{\prime}$. (Solid line) Empirical correlation $\left(r_{s}^{\prime} / r_{s}-1\right) \approx 6 \times 10^{-4}\left(L_{1} / r_{s}^{\prime}\right)^{17.0}$.

We will proceed now to the analysis of the position of the surface of tension for low curvature, including the flat phase interface. We chose a coordinate system $(z, \rho)$ with origin located within (or close to) the phase interface, at a distance $r_{x}$ from the center of the cluster. We substitute $r_{x}$ $+z$ for $r$ in the right-hand side of Eq. (A8), and $r_{x}+z_{s}^{\prime}$ for $r_{s}^{\prime}$ in the left-hand side,

$$
\left(r_{x}+z_{s}^{\prime}\right)^{3}=\frac{\int_{-r_{x}}^{\infty} c(\mathrm{~d} \rho / \mathrm{d} z)^{2}\left(r_{x}+z\right)^{2} \mathrm{~d} z}{\int_{-r_{x}}^{\infty} c(\mathrm{~d} \rho / \mathrm{d} z)^{2}\left(r_{x}+z\right)^{-1} \mathrm{~d} z} .
$$

In the denominator we use a Taylor series

$$
\left(r_{x}+z\right)^{-1}=r_{x}^{-1}\left[1+\sum_{k=1}^{\infty}(-1)^{k}\left(z / r_{x}\right)^{k}\right] \text {. }
$$

Series Eq. (A10) only converges for $|z|<r_{x}$. Therefore, we limit the integrations to the interval $\left(-r_{x}, r_{x}\right)$. This limitation is not restrictive as far as we consider sufficiently large clusters. Performing the integration on the members of the series and denoting $q_{x} \equiv 1 / r_{x}$, we obtain

$$
1+3 z_{s}^{\prime} q_{x}+3 z_{s}^{\prime 2} q_{x}^{2}+z_{s}^{\prime 3} q_{x}^{3}=\frac{1+2 s_{1} q_{x}+s_{2} q_{x}^{2}}{1+\sum_{k=1}^{\infty}(-1)^{k} s_{k} q_{x}^{k}},
$$

where

$$
s_{k} \equiv S_{k} / S_{0}, \quad S_{k} \equiv \int_{-r_{x}}^{r_{x}} c(\partial \rho / \partial z)^{2} z^{k} \mathrm{~d} z
$$

The position of the surface of tension $z_{s}^{\prime}$ can be obtained by solving Eq. (A11). For small curvatures we expand the solution in the form of a series,

$$
\begin{aligned}
z_{s}^{\prime}= & s_{1}+\left(-s_{1} s_{2}+\frac{1}{3} s_{3}+\frac{2}{3} s_{1}^{3}\right) q_{x}^{2} \\
& +\left(\frac{2}{3} s_{1} s_{3}-\frac{1}{3} s_{1}^{4}-\frac{1}{3} s_{4}\right) q_{x}^{3}+\cdots
\end{aligned}
$$

We see immediately that, for a flat phase interface, $z_{s}^{\prime}=s_{1}$, which is a proof of Eq. (32).
Further, we note that a term linear in curvature is missing. Therefore, the surface of tension remains close to the position given by Eq. (32) even for quite small clusters (high curvatures). This is a special feature of the radius of the surface of tension. The equimolar surface shifts from the planar position by a distance proportional to curvature. The observation that the surface of tension stays associated with the density profile was used to construct the approximate density profile of the cluster given by Eqs. (35) and (36).

\section{APPENDIX B: THERMOPHYSICAL PROPERTIES OF $n$-NONANE}

Surface tension. The surface tension for $n$-nonane has been measured by Jasper et al. ${ }^{38,54}$ Orthobaric $^{57}$ data $^{38}$ span over the range of $0-60{ }^{\circ} \mathrm{C}$ and has been correlated by the authors to a linear form $\sigma\left(\mathrm{mN} \mathrm{m}^{-1}\right)=24.84-0.09417(T / K$ $-273.15)$. Isobaric measurements ${ }^{54}$ were taken in a dry nitrogen atmosphere at a total pressure of 760 Torr $(101325 \mathrm{~Pa})$ in the range of $0-100{ }^{\circ} \mathrm{C}$. The data have been correlated by the authors as $\sigma\left(\mathrm{mN} \mathrm{m}^{-1}\right)=24.72$ $-0.09347(T / K-273.15)$. Adsorbed nitrogen decreases the surface tension. This effect is more pronounced at lower temperatures because the gas molecules with lower thermal energy adsorb more easily. Consequently, both the surface tension at $273.15 \mathrm{~K}$ and the slope have somewhat lower values for the isobaric case than for the orthobaric case. The determination of the influence parameter was based on the orthobaric data. ${ }^{38}$

Liquid density. Density of the liquid $n$-nonane was computed from the fundamental equation of state by Lemmon and Span. ${ }^{53}$ This equation provides a superior representation of density and other thermodynamic properties in both liquid and gas phases. The computed liquid densities are in a good agreement with earlier correlations. ${ }^{55}$

Vapor pressure. The vapor pressure of saturated $n$-nonane has been discussed in detail by Hung et al. ${ }^{24}$ These authors recommended equation by King and Al-Najjar, ${ }^{49}$ although a different correlation was used in a later study by Rudek et $a .^{27}$ More recently, a generalized vapor pressure equation has been obtained by Lemmon and Goodwin ${ }^{56}$ [their Eq. (12)]. Also, the vapor pressure can be computed from the fundamental equation of state by Lemmon and Span. ${ }^{53}$ We chose the correlation by King and Al-Najjar, which seems most safe for the necessary extrapolation to low temperatures.

Equation of state. The Peng-Robinson ${ }^{33}$ equation of state was used for the gradient theory computations. Accurate correlations of by Lemmon and Goodwin ${ }^{56}$ were used for the critical temperature, critical pressure, and the accentric factor of $n$-nonane [their Eqs. (20), (21), and (24), respectively].

\footnotetext{
${ }^{1}$ R. Becker and W. Döring, Ann. Phys. 24, 719 (1935).

${ }^{2}$ J. D. van der Waals, Z. Phys. Chem. (Leipzig) 13, 657 (1894).

${ }^{3}$ J. W. Cahn and J. E. Hilliard, J. Chem. Phys. 28, 258 (1958).

${ }^{4}$ J. W. Cahn and J. E. Hilliard, J. Chem. Phys. 31, 688 (1959).

${ }^{5}$ J. S. Rowlinson and B. Widom, Molecular Theory of Capilarity (Oxford University Press, Oxford, 1982).

${ }^{6}$ H. T. Davis, Statistical Mechanics of Phases, Interfaces, and Thin Films (VCH, New York, 1996).

${ }^{7}$ V. I. Kalikmanov, Statistical Physics of Fluids (Springer, Heidelberg, 2001).
} 
${ }^{8}$ V. G. Baidakov, S. P. Protsenko, G. G. Chernykh, and G. S. Boltachev, Phys. Rev. E 65, 041601 (2002).

${ }^{9}$ D. W. Oxtoby and R. Evans, J. Chem. Phys. 89, 7521 (1988).

${ }^{10}$ R. C. Tolman, J. Chem. Phys. 17, 333 (1949).

${ }^{11}$ A. Dillmann and G. E. A. Meier, J. Chem. Phys. 94, 3872 (1991).

${ }^{12}$ C. F. Delale and G. E. A. Meier, J. Chem. Phys. 98, 9850 (1993).

${ }^{13}$ A. Laaksonen, I. J. Ford, and M. Kulmala, Phys. Rev. E 49, 5517 (1994).

${ }^{14}$ V. I. Kalikmanov, J. Chem. Phys. 124, 124505 (2006).

${ }^{15}$ K. N. H. Looijmans, C. C. M. Luijten, and M. E. H. van Dongen, J. Chem. Phys. 103, 1714 (1995).

${ }^{16}$ C. C. M. Luijten, R. G. P. van Hooy, J. W. F. Janssen, and M. E. H. van Dongen, J. Chem. Phys. 109, 3553 (1998).

${ }^{17}$ C. C. M. Luijten, P. Peeters, and M. E. H. van Dongen, J. Chem. Phys. 111, 8335 (1999).

${ }^{18}$ P. Peeters, J. Hrubý, and M. van Dongen, J. Phys. Chem. B 105, 11763 (2001).

${ }^{19}$ D. G. Labetski, Ph.D. thesis, Eindhoven University of Technology, 2007.

${ }^{20}$ G. W. Adams, J. L. Schmitt, and R. A. Zalabsky, J. Chem. Phys. 81, 5074 (1984)

${ }^{21}$ P. E. Wagner and R. Strey, J. Chem. Phys. 80, 5266 (1984).

${ }^{22}$ Y. Viisanen, P. E. Wagner, and R. Strey, J. Chem. Phys. 108, 4257 (1998).

${ }^{23}$ J. L. Katz, J. Chem. Phys. 52, 4733 (1970).

${ }^{24}$ C.-H. Hung, M. J. Krasnopoler, and J. L. Katz, J. Chem. Phys. 90, 1856 (1989).

${ }^{25}$ C.-H. Hung, M. J. Krasnopoler, and J. L. Katz, J. Chem. Phys. 92, 7722 (1990).

${ }^{26}$ V. Ždímal, J. Smolfk, and I. G. Meijer, Collect. Czech. Chem. Commun. 59, 253 (1994).

${ }^{27}$ M. M. Rudek, J. A. Fisk, V. M. Chakarov, and J. L. Katz, J. Chem. Phys. 105, 4707 (1996).

${ }^{28}$ C. C. M. Luijten, Ph.D. thesis, Eindhoven University of Technology, 1998.

${ }^{29}$ R. M. Nyquist, V. Talanquer, and D. W. Oxtoby, J. Chem. Phys. 103, 1175 (1995)

${ }^{30}$ L. Gránásy, J. Chem. Phys. 104, 5188 (1996).
${ }^{31}$ L. Gránásy, J. Chem. Phys. 109, 9660 (1998).

${ }^{32}$ J. C. Barrett, J. Phys.: Condens. Matter 9, L19 (1997).

${ }^{33}$ D.-Y. Peng and D. B. Robinson, Ind. Eng. Chem. Fundam. 15, 59 (1976).

${ }^{34}$ N. F. Carnahan and K. E. Starling, J. Chem. Phys. 51, 635 (1969).

${ }^{35}$ J. Barrett, J. Chem. Phys. 107, 7989 (1997).

${ }^{36}$ S. Natarajan, S. A. Harris, and I. J. Ford, J. Chem. Phys. 124, 044318 (2006).

${ }^{37}$ A. Obeidat, J.-S. Li, and G. Wilemski, J. Chem. Phys. 121, 9510 (2004).

${ }^{38}$ J. J. Jasper, E. R. Kerr, and F. Gregorich, J. Am. Chem. Soc. 75, 5252 (1953).

${ }^{39}$ G. Wilemski and J.-S. Li, J. Chem. Phys. 121, 7821 (2004).

${ }^{40}$ V. Talanquer and D. W. Oxtoby, J. Chem. Phys. 100, 5191 (1994).

${ }^{41}$ J. Hrubý, Technical University of Eindhoven Research Report No. R-1501-D, 2000 (unpublished).

${ }^{42}$ R. K. Bowles, D. Reguera, Y. Djikaev, and H. Reiss, J. Chem. Phys. 115, 1853 (2001).

${ }^{43}$ W. Band, J. Chem. Phys. 7, 324 (1939).

${ }^{44}$ F. H. Stillinger, Jr., J. Chem. Phys. 38, 1486 (1963).

${ }^{45}$ D. W. Oxtoby and D. Kashchiev, J. Chem. Phys. 100, 7665 (1994).

${ }^{46}$ S. L. Girshick and C.-P. Chiu, J. Chem. Phys. 93, 1273 (1990).

${ }^{47}$ J. Hrubý, in Nucleation and Atmospheric Aerosols 2004, edited by M. Kasahara and M. Kulmala (Kyoto University Press, Kyoto, 2004), pp. $268-272$.

${ }^{48}$ D. Reguera and H. Reiss, J. Phys. Chem. B 108, 19831 (2004).

${ }^{49}$ M. B. King and H. Al-Najjan, Chem. Eng. Sci. 29, 1003 (1974).

${ }^{50}$ C. P. Bokis and M. D. Donohue, Ind. Eng. Chem. Res. 33, 146 (1994).

${ }^{51}$ B. K. Kaul and J. M. Prausnitz, Ind. Eng. Chem. Fundam. 16, 335 (1977).

${ }^{52}$ J. A. Fisk and J. L. Katz, J. Chem. Phys. 104, 8649 (1996).

${ }^{53}$ E. W. Lemmon and R. Span, J. Chem. Eng. Data 51, 785 (2006).

${ }_{55}^{54}$ J. J. Jasper and E. V. Kring, J. Phys. Chem. 59, 1019 (1955).

${ }^{55}$ M. J. Assael, J. H. Dymond, and D. Exadaktilou, Int. J. Thermophys. 15, 324 (1994).

${ }^{56}$ E. W. Lemmon and A. R. H. Goodwin, J. Phys. Chem. Ref. Data 29, 1 (2000).

${ }^{57}$ Orthobaric: Measured at the vapor pressure of the pure $n$-nonane. 